\title{
Indirect Methods for Constructing Radio Environment Map
}

by

Safwan Alfattani

Thesis submitted to the

Faculty of Graduate and Postdoctoral Studies

In partial fulfillment of the requirements

For the M.A.Sc. degree in

Electrical and Computer Engineering

School of Electrical Engineering and Computer Science

Faculty of Engineering

University of Ottawa

(C) Safwan Alfattani, Ottawa, Canada, 2016 
In the name of Allah, the Most Gracious, the Most Merciful 


\begin{abstract}
To solve the spectrum scarcity problem caused by the high number of wireless applications and users, the concept of cognitive radio (CR) was proposed in the past few years. Cognitive radio networks (CRNs) provide dynamic spectrum access (DSA), where the unlicensed users can access the spectrum without causing unacceptable level of interference to the primary user (PU). DSA was based on conventional spectrum sensing information or geolocation databases. Later, radio environment map (REM) as an improved geolocation database was introduced to enhance the DSA process. It is a comprehensive map consists of different integrated databases, and the interference field information is one of its databases.

In this thesis, a description of the REM concept and its construction methods will be presented. The focus will be for the indirect methods for constructing interference map, which represents a layer of the REM. Indirect method refers to the methods that utilize known model information, to first estimate the primary transmitter parameters and then generate REM. Two indirect methods under lognormal shadowing were presented and compared. The better of these two methods is further investigated in different scenarios. These scenarios include different number of sensors, varied size of measurements, several shadowing spread values, different percentages of error in path-loss exponent, and the effect of the number of moving sensors and their speeds to the REM quality. The performance is evaluated using these metrics: "localization error, signal power error and correct detection zone ratio (CDZR). The results show that performance is enhanced as the number of sensors and the size of measurements increase, whereas clear degradation in REM quality is shown when shadowing spread increases or the model parameters are not well calibrated. Also, as the number of moving sensors or their speeds increase, the REM performance becomes less effective.
\end{abstract}




\section{Acknowledgments}

Writing this thesis has been a period of intense learning for me, not only in the scientific field, but also on a personal level. After thanking Allah the Almighty, I would like to express my sincere gratitude to all those who are behind my success and supported me throughout this period.

First and foremost, my deepest appreciation goes to my supervisor Prof.Abbas Yongacoglu for his continuous support and invaluable guidance during my study. His great experience and technical knowledge regarding the research direction made my journey much easier. Prof.Abbas always shows me his support and understanding and his office's door was always open whenever I ran into a trouble spot or had a question about my research. Without his supervision or constant help, this thesis would not have been possible. I feel very privileged being supported by such a kind professor.

I would also like to acknowledge with much appreciation the crucial role of my parents who encouraged me and supported me by their effort and prayers.

Finally, I would also like to thank my wife for her support, care, patience and encouragement throughout my study. I would like to give special thanks to my daughter (who was born during my Master's program) for her love and being patient for the whole duration. 


\section{Table of Contents}

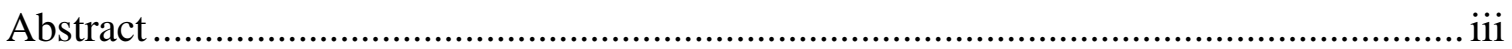

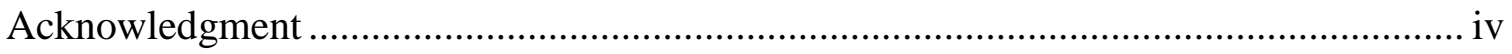

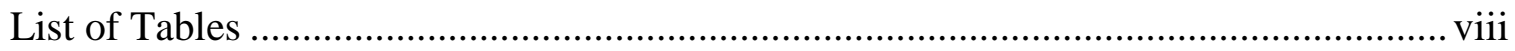

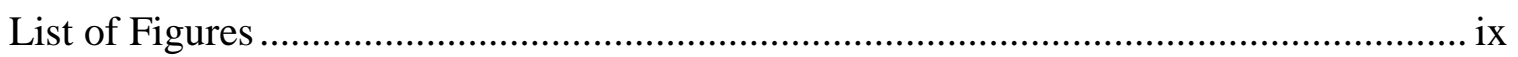

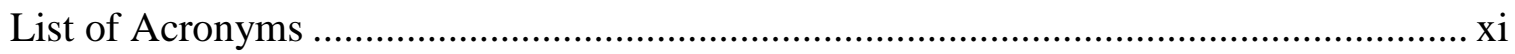

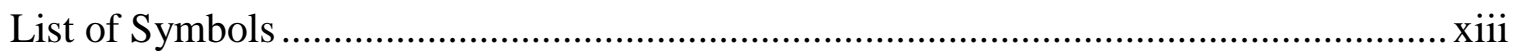

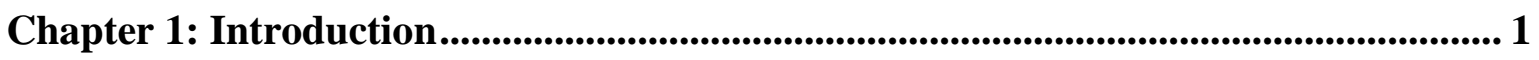

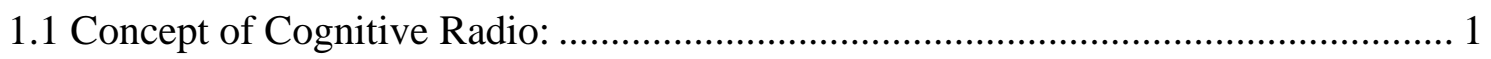

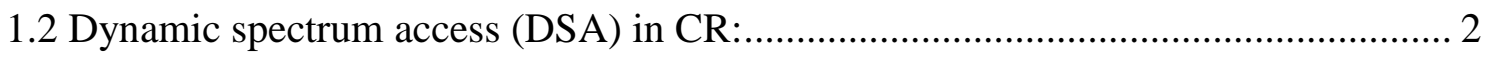

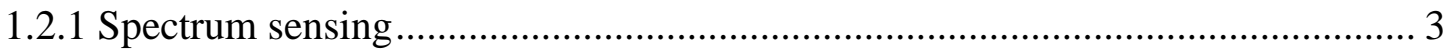

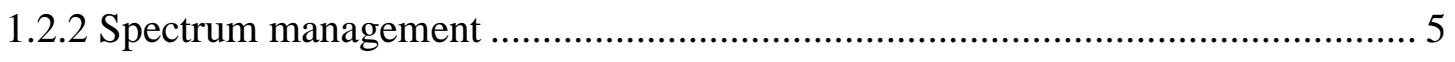

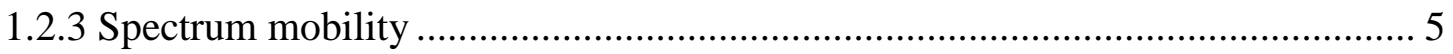

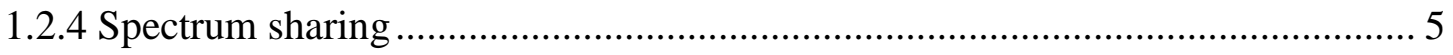

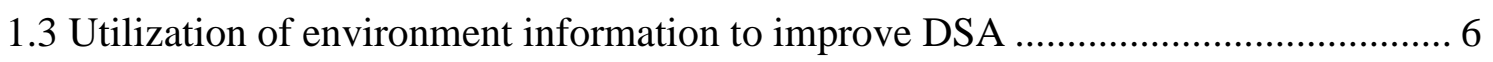

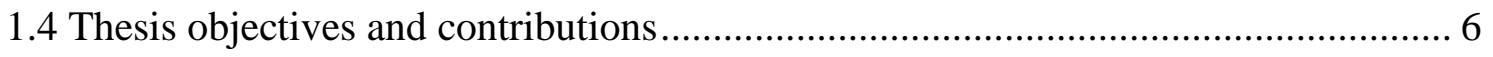

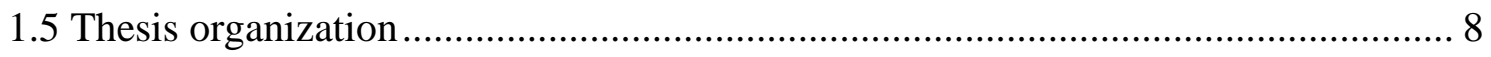

Chapter 2: Radio Environment Map (REM) .......................................................... 10

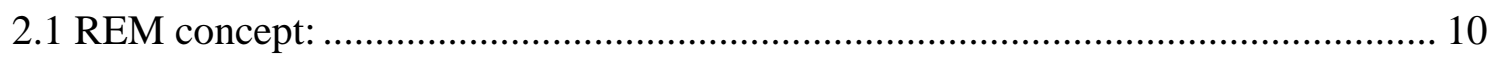

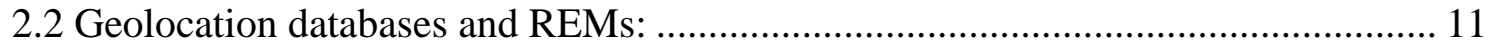

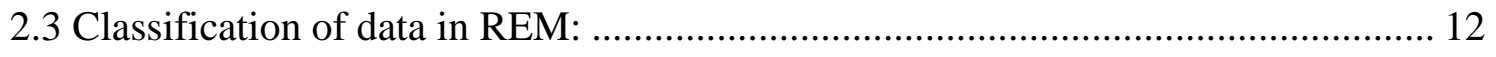

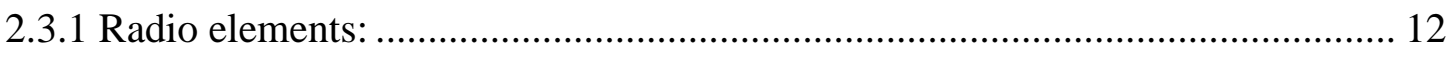

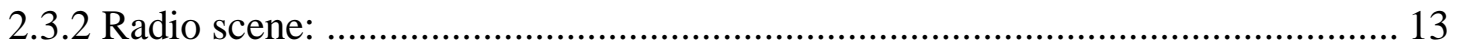

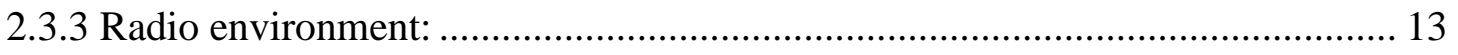

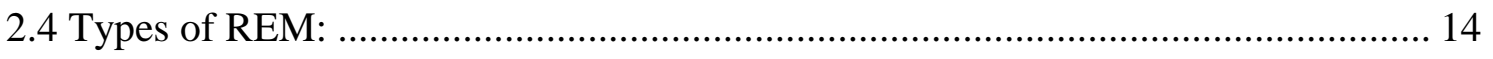

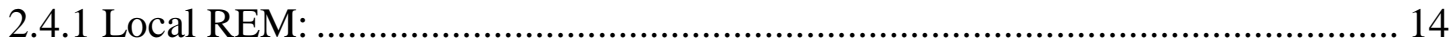




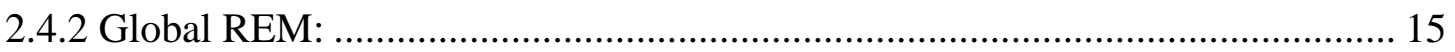

2.5 Approaches of situation awareness in REMs: .............................................. 15

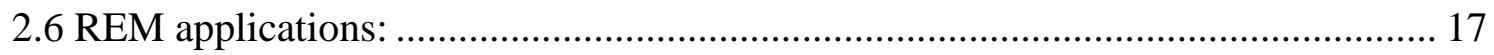

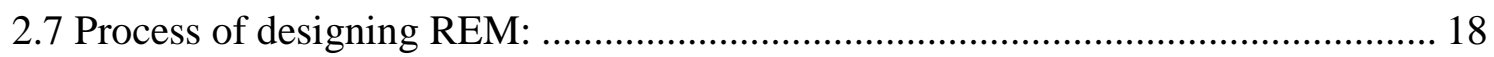

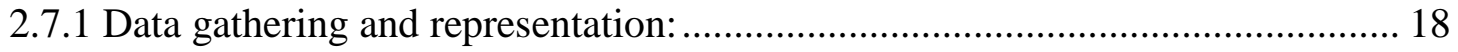

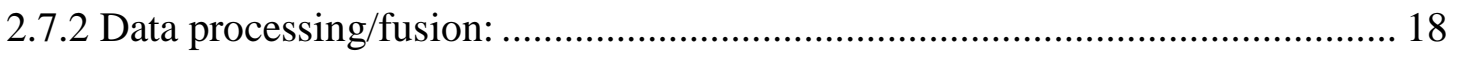

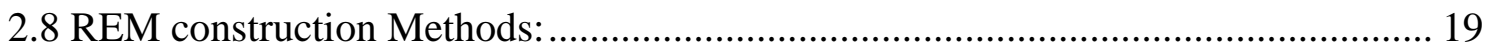

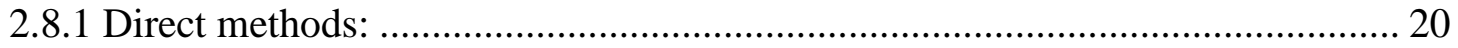

2.8.1.1 Techniques for direct methods:...................................................... 20

2.8.1.2 Comparative studies of direct methods:............................................ 23

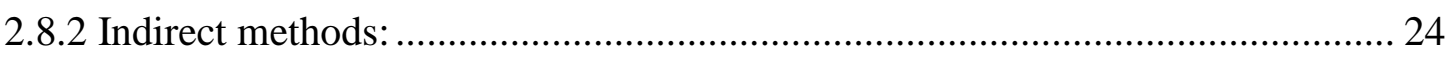

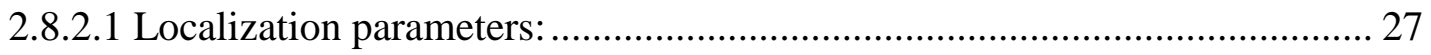

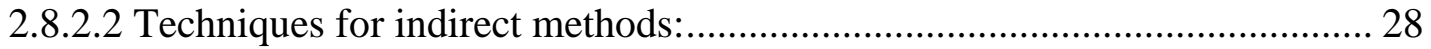

2.8.2.3 Comparative studies of indirect methods:............................................... 29

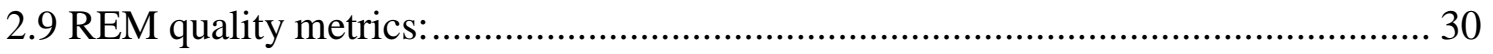

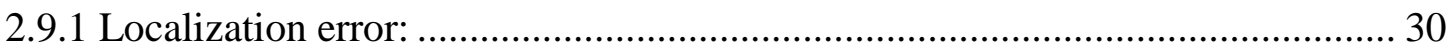

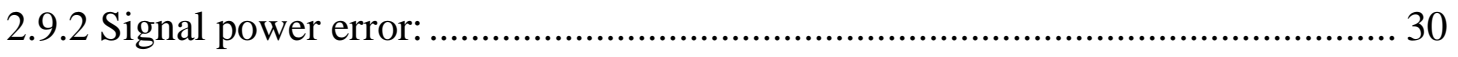

2.9.3 Correct Detection Zone Ratio and False Alarm Zone Ratio: ........................... 30

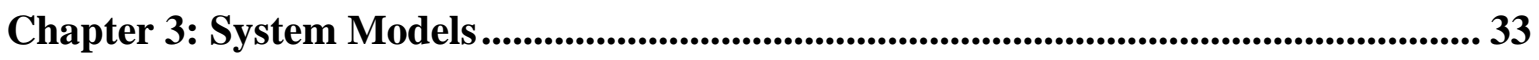

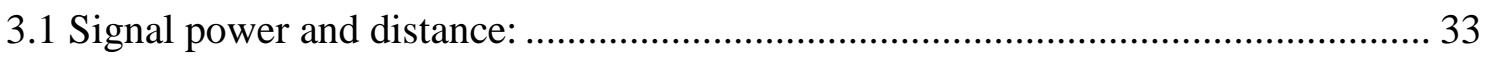

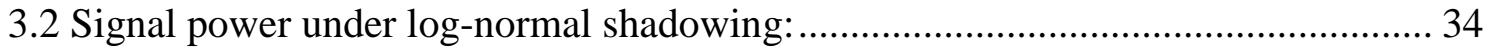

3.3 Mathematical description of some indirect methods to construct REM: ............... 35

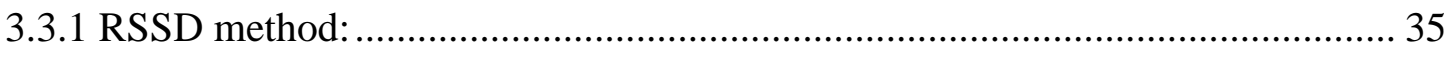

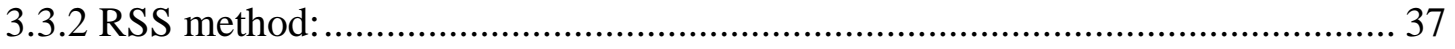

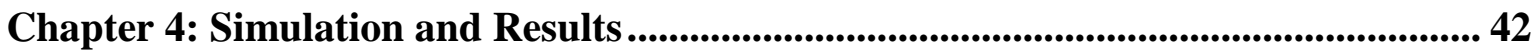

4.1 Comparison between RSSD and RSS methods: ............................................ 42

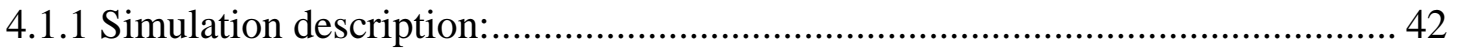

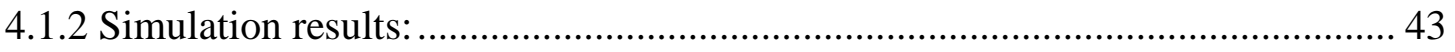

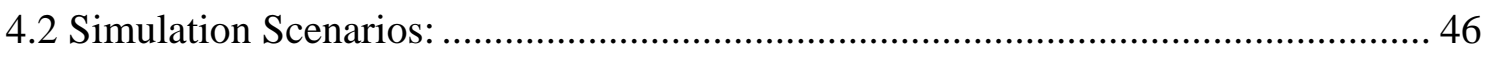


4.2.1 Scenario 1: (performance test with different number of sensors) 46

4.2.2 Scenario 2: (performance test with different size of hypothetical measurements) 49

4.2.3 Scenario 3: (performance test under different shadowing spread values) ......... 53

4.2.4 Scenario 4: (performance test with error in path-loss exponent)...................... 55

4.2.5 Scenario 5: (REM performance with some moving sensors by a constant speed) 58

4.2.6 Scenario 6: (REM performance with some moving sensors by different speeds)61

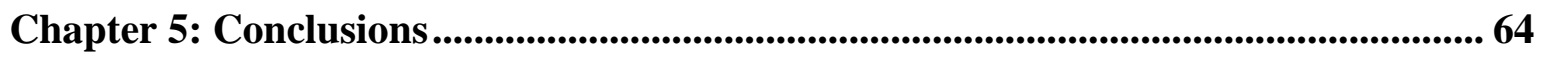

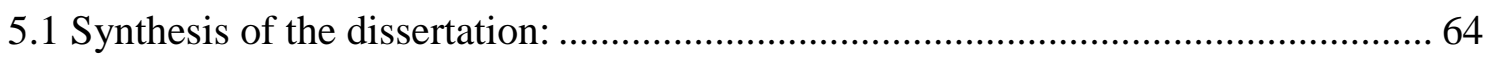

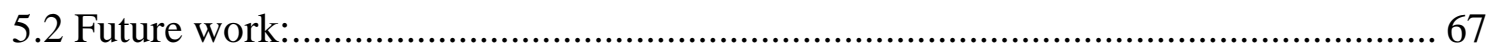

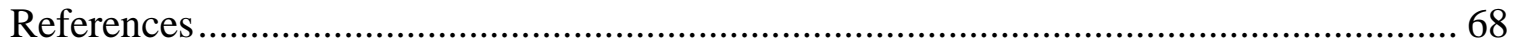




\section{List of Tables}

Table 1: Path-loss Exponents for Different Environments ................................................. 38

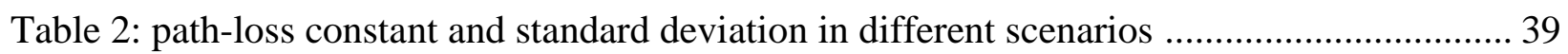

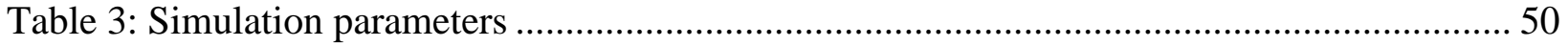




\section{List of Figures}

Figure 1-1: Utilization of spectrum holes …...................................................................... 3

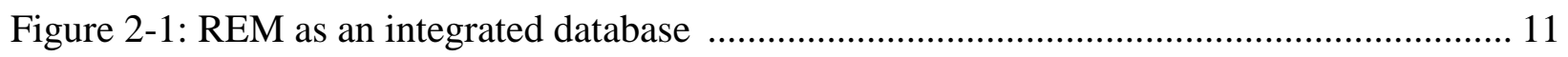

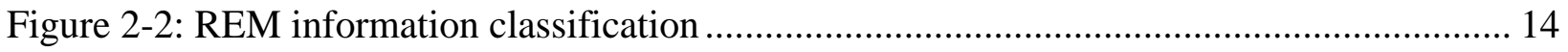

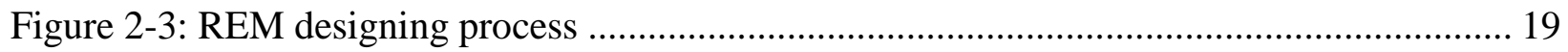

Figure 2-4: The difference between A) direct method and B) indirect method for constructing

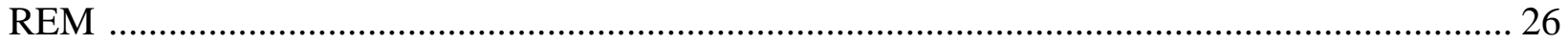

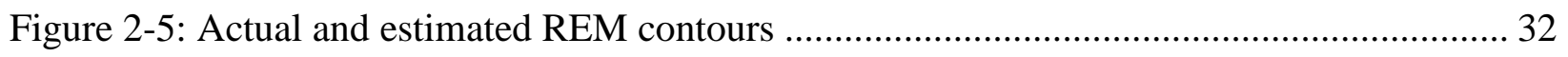

Figure 4-1: Distribution of sensors around the primary user transmitter................................ 43

Figure 4-2: Performance comparison between RSS and RSSD algorithms ............................ 44

Figure 4-3: Number of sensors versus error of the estimated transmitted power ...................... 47

Figure 4-4: Number of sensors versus correct detection zone ratio (CDZR) .......................... 48

Figure 4-5: Distribution of sensors around the primary user .......................................... 50

Figure 4-6: Number of measurements versus error of the transmitter location ......................... 51

Figure 4-7: Number of measurements versus error of the transmitted power ......................... 51

Figure 4-8: Number of measurements versus correct detection zone ratio (CDZR) .................. 52

Figure 4-9: Shadow fading standard deviation versus error of the transmitter location.............. 54

Figure 4-10: Shadow fading standard deviation versus error of the transmitted power .............. 54

Figure 4-11: Shadow fading standard deviation versus CDZR .......................................... 55

Figure 4-12: Localization error for different percentages of error in $(\alpha)$ and different number of

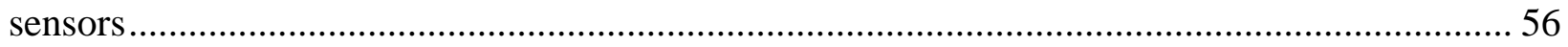

Figure 4-13: CDZR for different percentages of error in $(\alpha)$ and different number of sensors .... 57 
Figure 4-14: Distribution of fixed and mobile moving sensors around the primary user transmitter.......

Figure 4-15: Performance comparison for different number of moving sensors versus transmitter

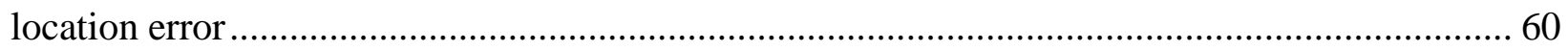

Figure 4-16: Performance comparison of different speeds of moving sensors ........................ 62 


\section{List of Acronyms}

$\begin{array}{ll}\text { AOA } & \text { Angle of Arrival } \\ \text { AP } & \text { access point } \\ \text { ARM } & \text { Available Resource Map } \\ \text { BS } & \text { Base Station } \\ \text { CDZR } & \text { Correct Detection Zone Ratio } \\ \text { CR } & \text { Cognitive Radio } \\ \text { CRN } & \text { Cognitive Radio Network } \\ \text { DSA } & \text { Dynamic Spectrum Access } \\ \text { FAZR } & \text { False Alarm Zone Ratio } \\ \text { FCC } & \text { Federal Communications Commission } \\ \text { GIDS } & \text { Gradient plus Inverse Distance Squared } \\ \text { GIS } & \text { Geographical Information System } \\ \text { GPS } & \text { Global Positioning System } \\ \text { IC } & \text { Interference Cartography } \\ \text { IDW } & \text { Inverse Distance Weighted } \\ \text { IEEE } & \text { Institute of Electrical Engineering } \\ \text { LAN } & \text { Local Area Network } \\ \text { LOS } & \text { Line-of-Sight } \\ \text { LTE } & \text { Long Term Evolution } \\ \text { MCD } & \text { Measurement Capable Device } \\ \text { MH } & \text { Metropolis-Hastings } \\ \text { MSM } & \text { Modified Shepard's Method } \\ \text { PLOS } & \text { Personal Digital Assistant } \\ \text { PDe-of-Sight }\end{array}$




$\begin{array}{ll}\text { PU } & \text { Primary User } \\ \text { QoS } & \text { Quality of Service } \\ \text { RBF } & \text { Radial Basis Functions } \\ \text { REM } & \text { Radio Environment Map } \\ \text { RF } & \text { Radio Frequency } \\ \text { RMSE } & \text { Root Mean Squared Error } \\ \text { RSS } & \text { Received Signal Strength } \\ \text { RSSD } & \text { Received Signal Strength Difference } \\ \text { RSSI } & \text { Receiver's Received Signal Strength Indicator } \\ \text { Rx } & \text { Received signal } \\ \text { SDR } & \text { Software-Defined Radio } \\ \text { SNR } & \text { Signal-to-Noise-Ratio } \\ \text { SU } & \text { Secondary User } \\ \text { TOA } & \text { Time of Arrival } \\ \text { Tx } & \text { Transmitted signal } \\ \text { UHF } & \text { Ultra-High Frequency (300 MHz - 3 GHz) } \\ \text { UWAN } & \text { Unlicensed Wide Area Network } \\ \text { VHF } & \text { Very High Frequency (30 MHz - 300 MHz) } \\ \text { WLAN } & \text { Wireless Local Area Network } \\ \text { WRAN } & \text { Wireless Regional Area Network } \\ & \end{array}$




\section{List of Symbols}

$\mathrm{Z}_{1}{ }^{\mathrm{CD}} \quad$ Correct detection zone for the active transmitter

$\mathrm{Z}_{0}{ }^{\mathrm{CD}} \quad$ Correct detection for free transmission zones

$\mathrm{Z}^{\mathrm{FA}} \quad$ False alarm zone

$Z^{\mathrm{MD}} \quad$ Missed detection zone

$A(Z) \quad$ Area of the zone

$d_{0} \quad$ Reference distance which is selected in accordance with propagation environment

$\overline{P L}\left(d_{0}\right) \quad$ Reference path-loss obtained distance $d_{0}$

$G_{t} \quad$ Transmitter antenna gain

$G_{r} \quad$ Receiver antenna gain

$\lambda \quad$ Wavelength of the RF signal in meters

$\alpha \quad$ Path-loss exponent

$\overline{P L}(d) \quad$ Average path-loss

$\sigma \quad$ Standard deviation for the shadowing effect on the RF signal

$P_{r}(d) \quad$ Received signal at distance $(d)$

$P_{t} \quad$ Power of the transmitted signal

$d_{k} \quad$ Distance between the primary user transmitter and a sensor located at $\left(x_{k}, y_{k}\right)$

$P_{k m} \quad$ Power difference between two sensors located at $\left(x_{k}, y_{k}\right)$ and $\left(x_{m}, y_{m}\right)$

$\left(\widehat{x_{p u}}, \widehat{y_{p u}}\right) \quad$ Estimated location of the primary user transmitter

$l_{0} \quad$ Path-loss constant 


\section{Chapter 1: Introduction}

\subsection{Concept of Cognitive Radio:}

Development of communication technologies is one of the fundamental aspects of the modern societies. This is due to the large dependency of everyday life applications on data communication networks including education, shopping, entertainment and social interactions. This evolution has led to the software-defined radio (SDR) technology, where the communication system components and the process of signals are entirely implemented in software instead of typical implementation in hardware.

Although the ease and speed of software-defined radio systems have opened the door for plenty of communication applications, they are not capable of reconfiguring their operating parameters into the most effective form based on changing conditions and requirements. A newer improved version of SDR is the cognitive radio (CR), which was envisioned by Joseph Mitola III in 1999 [1]. He defined the (CR) term as "The point at which wireless personal digital assistants (PDAs) and the related networks are sufficiently computationally intelligent about radio resources and related computer-to-computer communications to: a) detect user communications needs as a function of use context, and b) to provide radio resources and wireless services most appropriate to those needs" [2].

Since Mitola's dissertation, many research papers have been published to explore the capabilities of this new concept, and different definitions have been suggested. For example, Haykin conceived the CR as a smart system which is aware of its surroundings and can learn from the environment and adapt to the RF changes by corresponding changes in its operating parameters to achieve two goals: highly reliable communication and efficient 
spectrum utilization [3]. FCC defines $\mathrm{CR}$ as "a radio that can change its transmitter parameters based on interaction with the environment in which it operates. This interaction may involve active negotiations with other spectrum users and/or passive sensing and decision making (smart radio) within the radio. The majority of CRs will probably be SDRs, but a CR does not necessarily use software, nor does it need to be field programmable".

In spite of the differences between those definitions, two main features are common in CR definitions. They are smart cognition and re-configurability.

Smart cognition means the ability to learn about the surrounding environment and act accordingly. For example, it has the ability to analyze sensors data and, learns the interference levels, detects the spectrum holes and obtains the RF map. It is also capable of recognizing transmitter patterns and obtains their parameters (frequency, power, time, location).

Re-configurability means that $\mathrm{CR}$ has the flexibility of changing its operational parameters based on the new changes of the surrounding environment. It may change operating frequency, modulation scheme and transmitted power as the conditions and requirements change.

\subsection{Dynamic spectrum access (DSA) in CR:}

One of the main applications of CR networks is to solve the spectrum scarcity problem. This is caused by the increasing demand for bandwidth, due the excessive amount of wireless applications and users. The electromagnetic radio spectrum is a natural resource, whose usage is controlled by governmental licences. Instead of restricting the frequency band for licensed users, the idea of allowing unlicensed users (secondary users) to use the 
spectrum without disturbing licensed users (primary users) is called dynamic spectrum access.

Recent studies of spectrum occupancy measurements in different countries reveal that large bands of the spectrum have low utilization, either lightly used or totally unused [4],[5]. These bands can be considered as spectrum holes, which can be defined as " $a$ band of frequencies assigned to a primary user, but, at a particular time and specific geographic location, the band is not being utilized by that user [3]”. The spectrum hole is also called as a white space. Cognitive radio networks (CRNs) are capable of utilizing these spectrum holes or white spaces to allow secondary users access the spectrum (Figure 1-1).

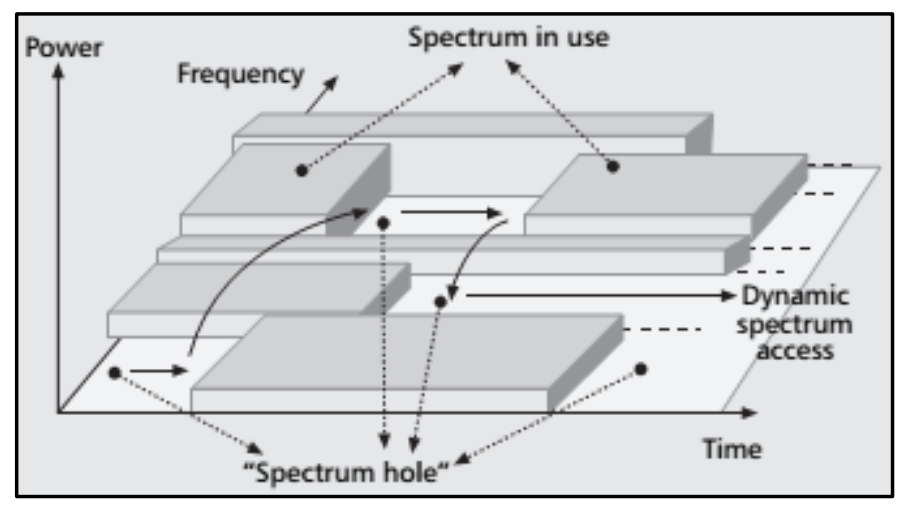

Figure 1-1: Utilization of spectrum holes [6]

There are four main steps in dynamic spectrum process. They are spectrum sensing, spectrum management, spectrum mobility, and spectrum sharing [7]. They will be described briefly as follow:

\subsubsection{Spectrum sensing}

Spectrum sensing is the first step in DSA process, and its objective is to detect the primary users and find the possible opportunities in the spectrum for secondary users. Sensing the spectrum should be done frequently because a frequency band that was unoccupied at a certain time instant could be occupied at a subsequent time instant. 
Detecting spectrum opportunities is done by detecting primary transmitters, and it is the responsibility of secondary users. Generally, detecting the primary transmitter can be classified in two categories, non-cooperative approaches and cooperative approaches.

Non-cooperative approaches refer to methods where no cooperation between secondary users to take decisions and they include these known methods: energy detector, cyclostationary detector, and matched filter. Energy detection is implemented by comparing the received signal energy with a predefined energy threshold to detect the presence of a primary user [8], while cyclostationary detection utilizes the periodic features of the received signals for better detection of primary signals under low signal-to-noise-ratio (SNR) conditions [9],[10]. Matched filter is the optimal detector when a complete prior knowledge of the primary user transmitted power is available and known to the secondary users [11]. Detailed comparisons between these detection techniques are discussed in various references [7],[12].

The performance of previous single detectors can be severely degraded because of fading, shadowing and errors in sensor measurements. An improved performance could be achieved if cooperation between secondary users is utilized [13]. There are two common methods of cooperative sensing detection: centralized and distributed cooperation [14]. However, generally cooperative sensing is implemented in three steps [15]. First, each secondary user or sensor node senses the spectrum independently to decide whether the frequency band of interest is used by a primary user or not. Second, the independent decision of each secondary user will be combined by a central decision maker which could be an access point (AP) in a wireless LAN or a base station (BS) in a cellular network. 
Third, the decision maker will make a final decision based on a decision rule about the presence or absence of a primary user.

\subsubsection{Spectrum management}

After detecting the free bands for usage by secondary users, the second step is to select the best channel to meet user communication requirements. This selection is made by first analyzing the spectrum holes and defining their characteristics. These characteristics include: channel bandwidth, operating frequency, interference level, channel error rate and primary user activity periods. Then, after obtaining all the spectrum properties, based on a decision rule, the best spectrum band that meets the QoS requirements will be chosen for secondary users [7].

\subsubsection{Spectrum mobility}

The goal of this step is to provide seamless transition for secondary users to another channel when a primary user appears or current channel conditions become worse. When spectrum handoff and switching to another channel happens, the operating frequency will be changed and other parameters will be adapted to the new channel. This operation should be done smoothly and quickly.

\subsubsection{Spectrum sharing}

The purpose of this step is to coordinate between secondary users to access the available spectrum bands. Spectrum sharing techniques can be assorted in three categories. They can be classified based on the architecture, which can be characterized as distributed 
spectrum sharing or centralized spectrum sharing. Also, they can be categorized according to spectrum allocation behavior as cooperative spectrum sharing or non-cooperative spectrum sharing. The third category for spectrum sharing techniques is based on the spectrum access technology, which can be overlay or underlay spectrum sharing [7].

\subsection{Utilization of environment information to improve DSA}

Much of the research on dynamic spectrum access in cognitive radio networks has been focused on obtaining the available spectrum opportunities and related environmental information only at the time of decision making. However, several recent studies show that acquiring relatively long-term knowledge of additional environmental information can enhance the performance of DSA. Examples of the acquired information include interference level, geographic terrain, access rights and transmitters' behavior. Obtaining such information can be accomplished by a sort of cooperative sensing, which was briefly explained in Section 1.2.1 This information is supposed to be stored in a kind of a database system, which might be consulted when a secondary user wants to access the spectrum. Building such knowledge about the radio environment to improve DSA is called radio environment map (REM), which will be further explored throughout this thesis.

\subsection{Thesis objectives and contributions}

This thesis aims to explore the indirect methods for REM construction in CRNs. Direct REM construction methods refer to methods that utilize measured values of signal power at sensing nodes to generate an estimated interference map at the area of interest by using different interpolation techniques, whereas the indirect construction methods use the 
sensing information to estimate the location of the transmitter and its associated parameters, and then by applying this information in a valid model, REM can be established.

Two indirect techniques will be explained in details and compared in terms of transmitter localizing the accuracy. In order to study the influential factors on REM performance using indirect methods, the technique that shows better results using localization metric will be further examined in different scenarios. These scenarios include different number of sensors and varied sample size of measurements. In addition, the effect of shadowing spread values and sensitivity of path-loss exponent values to the accuracy of generated REM will be investigated. Furthermore, the impact of sensors' mobility feature and their speed to REM performance will be tested in two scenarios. The first one examines different numbers of moving sensors with constant speed, while the other one assesses the performance with different speeds of moving sensors.

The main contributions of this thesis are threefold:

- Comparing between two indirect methods for constructing REM in terms of transmitter localization error.

- Studying the important factors that affect the REM accuracy. The results show performance improvement as the number of sensors or size of measurements increases. But, if the environment is subjected to high shadow fading, the accuracy of the estimated REM will decrease. Also, considering mobility of the SUs affects the REM quality negatively.

- The direct interpolation techniques might be better than the indirect techniques when the model parameters have high tolerance percentages or if we do not have good estimation of the propagation model. 


\subsection{Thesis organization}

The following is an overview of the remaining chapters of the thesis:

- Chapter 2: Radio Environment Map (REM) - This chapter starts by describing the REM concept, and explains the differences between geolocation database and REMs. It also clarifies the different classes of stored data in REMs and illustrates the two types of REMs local and global. In addition, a general description of approaches to acquire different REM information and the possible application scenarios for REMs in CRNs are presented. Moreover, this chapter explains the process for designing REM. It also describes two types of REM construction methods, (direct and indirect). For the direct methods, a brief commentary of some well-known direct techniques and some comparative studies between them will be presented. Since indirect methods are based on localization techniques, some common localization parameters are introduced. Also, several techniques and comparative studies of indirect construction methods are mentioned. The chapter is concluded by discussing the metrics used for evaluating the performance of constructed REMs.

- Chapter 3: System Models - This chapter presents two indirect techniques for constructing REM. The first technique is based on RSSD parameter while the second one is based on RSS. The chapter begins by describing the log-normal shadowing model, which represents the propagation model of the considered environment. Then, detailed mathematical analyses of these indirect techniques are explained. 
- Chapter 4: Simulation and Results - The goal of this chapter is to discuss the performance of indirect methods under different circumstances. First, performance comparison between RSSD method and RSS method is conducted. Then, the RSS technique is selected for further investigations in different scenarios. These scenarios include different number of sensors, varied size of measurements, different shadowing spread values and various percentages of error in estimated path-loss exponent. Furthermore, the mobility feature of sensors is examined in two scenarios. The first one tests the performance of different number of moving sensors with constant speed, whereas the second one evaluates the performance for moving sensors with varied speeds.

- Chapter 5: Conclusion - This chapter summarizes the dissertation and outlines the main derived results. It also proposes some ideas for future research directions. 


\section{Chapter 2: Radio Environment Map (REM)}

\subsection{REM concept:}

The Radio Environment Map (REM) concept can be considered as an extension to the available resource map (ARM) notion, which was described in [16] as a real time map of all radio activity in the network for cognitive radio applications in unlicensed wide area networks (UWANs). REM term is a newer advanced version that has more types of information and larger size of possible applications. It was first introduced by Zhao in his paper [17] and PhD thesis [18] as an approach to develop cognitive radios. It was envisioned as an abstraction of real-world radio environment, which sorts the multiple domains of cognitive radio networks, such as geographical features, regulation, policy, locations and activities of neighboring radios and radio frequency (RF) emissions, and stores it in an integrated database. The database information should be updated frequently to keep the REM information current, by taking frequent measurements and observations from the sensing nodes. The REM as an integrated database is depicted in Figure 2-1, where each layer of REM represents a different kind of database.

The REM can support cognition functionality, such as situation awareness, planning, reasoning and decision making. Furthermore, by using REM, the performance of CRNs can be remarkably enhanced in terms of adaption time, average packet delay and mitigation of hidden terminal problem [19]. 


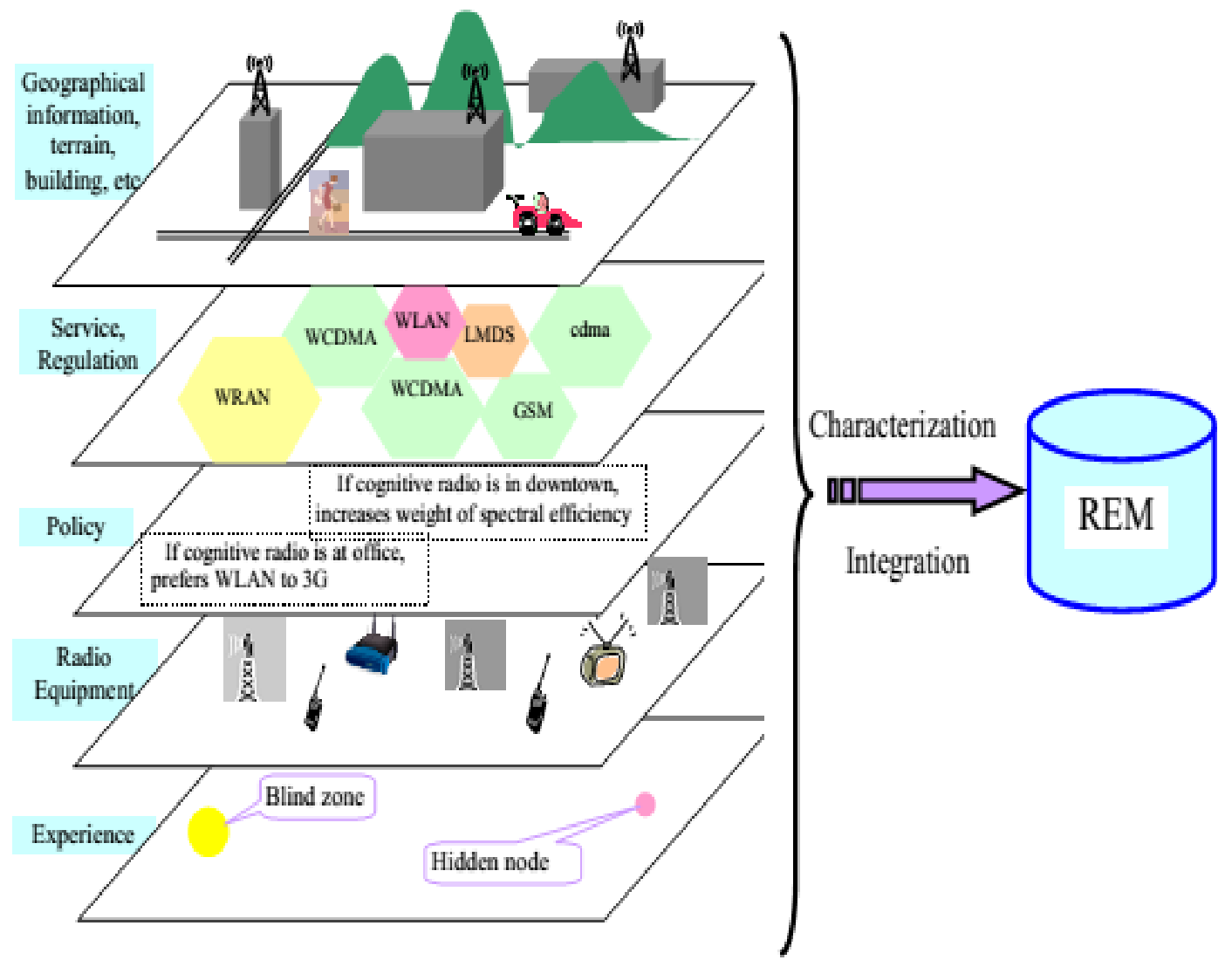

Figure 2-1: REM as an integrated database [18]

\subsection{Geolocation databases and REMs:}

One of the methods to find spectrum opportunities for secondary users, is to consult a database that has a list of available frequencies at each location [13]. In fact, new rules require unlicensed users to contact authorized database to find a list of available channels for communication [20]. Nowadays, there are many websites that contain spectrum database and provide available channels in the TV band at any location in the US or Canada ${ }^{1}$. By consulting

\footnotetext{
${ }^{1}$ https://www.google.com/get/spectrumdatabase/, http://whitespaces.spectrumbridge.com/whitespaces/home.aspx , https://spectrumbridge.com/tv-white-space/ , http://whitespaces.msresearch.us/ and http://www.tvfool.com/
} 
these databases, we can explore the location of transmitters, interference level, and available channels for secondary users.

However, conventional geolocation databases show some limitations in highly dynamic radio environment. REM is a sort of improved version, which combines geolocation databases with spectrum sensing methods. It uses the sensing techniques in a cooperative manner to build integrated databases which contain various useful types of information in order to find the available spectrum opportunities and make the DSA process more efficient. Moreover, REM scans the whole spectrum and detects any primary transmitters in the RF range, not necessarily TV transmitters in TV bands, as in most geolocation databases. Besides, REM contains different databases for different kinds of information, and one of them is the geolocation information [18]. In addition, REM is updated frequently to become more suitable for dynamic frequency map, which can capture the frequency bands that are only occasionally in use. Types of information contained in REM are described in the next section.

\subsection{Classification of data in REM:}

In the literature, the information contained in the REM is classified into three categories [21]. These categories are radio elements, radio scene and radio-environment characteristics. The type of the information stored within each category is summarized and depicted in Figure 2-2.

\subsubsection{Radio elements:}

This category keeps all the information related to the radio devices. The devices include transmitters, receivers and transceivers, and they cover a wide range of devices, from large basestations to small cell phones or sensors. This category also contains information that describes 
the $\mathrm{Tx} / \mathrm{Rx}$ operation which includes the details of the transmitted and received signal wave. In addition, information related to the location and mobility of the devices in the area of interest is stored under this category.

\subsubsection{Radio scene:}

This category retains all the information that regulates the behavior of the radio devices in the interested area. This includes policies, network operators and security and access rights. This category contains also information about the protocols that organize the communication between users' applications and services provided by REM.

\subsubsection{Radio environment:}

This category contains all the information related to the characteristics of radio environment. This includes the propagation models and the geographical terrain properties that affect the accuracy of the propagation models. Also, this category contains the most important information of the REM, which is the radio interference field estimation in a specific area. 


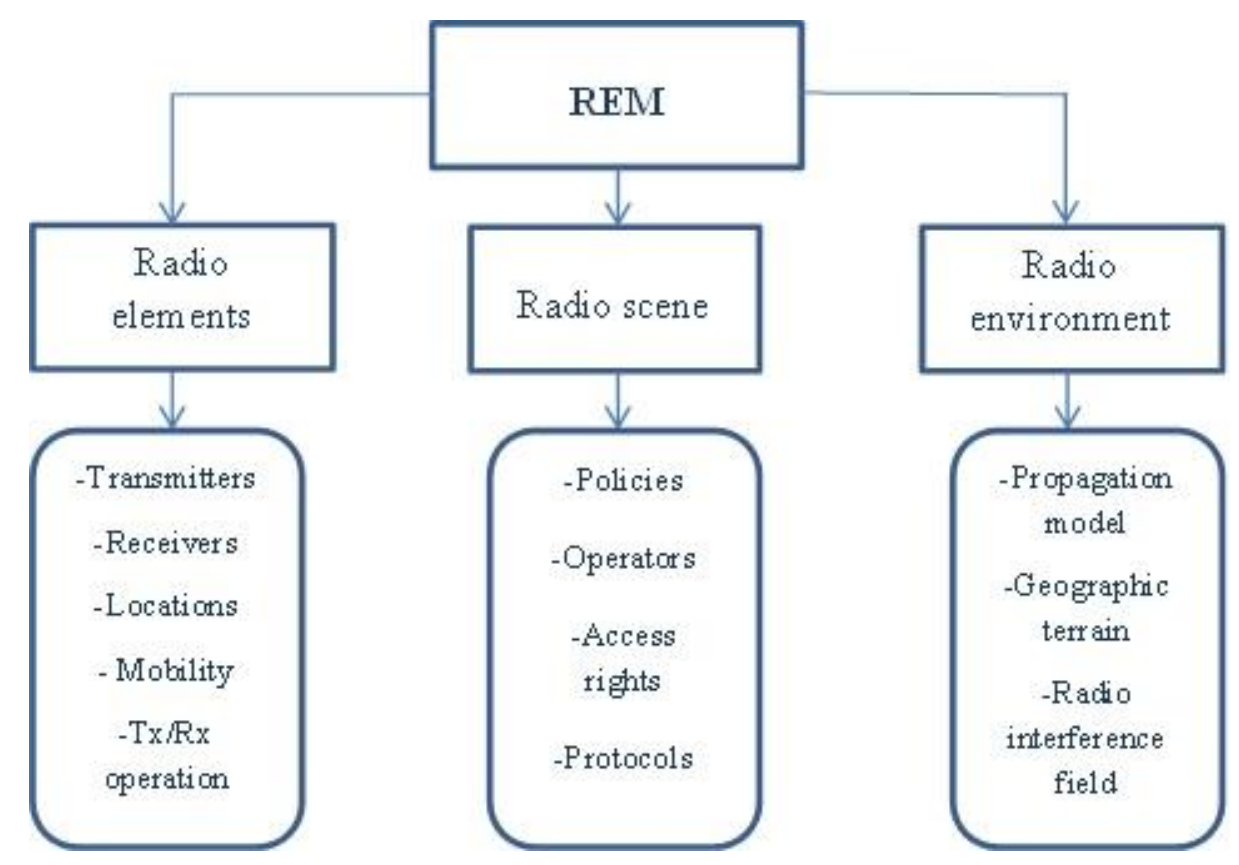

Figure 2-2: REM information classification

\subsection{Types of REM:}

In cognitive radio networks (CRNs), there are two types of REM; local and global REMs. Both of them may exchange information in a collaborative manner in order to get better and efficient DSA [17]:

\subsubsection{Local REM:}

It can exist at the user's terminal equipment and may be unique to each user's device. Its scale is determined by the unit service area, e.g., a cell for a cellular network [22]. A local REM can be used by each cognitive radio device to keep a record of its past experience and current status. In addition, local REMs can be used in ad hoc mesh networks that consist of cognitive radios to improve the communication infrastructure [17].

The local REM may synchronize and exchange information with the global REM through some common control channel. By sharing or exchanging the local REMs, node and channel 
awareness can be realized and cognitive radio device may learn to match the right routing protocol.

\subsubsection{Global REM:}

It exists at the network level providing wide processing capabilities, and its scale depends on the size of network's service area [17],[22].

The global REM has an essential job in many infrastructure based radio systems, such as the IEEE 802.22 wireless regional area network (WRAN) and cellular radio systems to help the wireless regional area network base station (WRAN BS) to know the location and transmitted power of nearby TV stations and determine the best spectrum opportunity to use [17]. Also, the global REM can be used in cognitive wireless local area network (WLAN) to support networkbased interference management [17].

\subsection{Approaches of situation awareness in REMs:}

Obtaining situation awareness is one of the most important features of CRNs. Unlike conventional sensing techniques, REMs as described previously contain multi domain information that yields complete efficient situation awareness. In this section, a brief description of possible approaches to acquire awareness of some different kinds of information in REMs is introduced.

- Location awareness: Transmitters' and receivers' location information either in the form of latitude, longitude and altitude or relative to some reference nodes should be known or acquired by REM. This can be approached by several localization techniques, Global Positioning 
System (GPS), or location databases. Getting location awareness might be by a combination of these approaches.

- Geographical information: knowing the terrain information of a radio environment is crucial for accurate modeling and better configuration of a CR communication system. It plays a major role in determining path loss and shadowing spread values. This awareness can be accomplished by a geological site survey or some terrain prediction and recognition techniques. In addition, available Geographical Information System (GIS) databases can be utilized to infer the geographical awareness ${ }^{2}$.

- RF Interference awareness: REM should obtain the interference level in the radio environment and all related information. This knowledge is extremely important, as it will detect the existence of primary users and decide the availabilities of channels for secondary users. Obtaining this awareness can be carried out through collective observations by CR users or direct field measurements by sensing nodes. Spectrum utilization databases may also help in getting this awareness.

- Mobility awareness: Information about mobile users and their speed and direction can be obtained or estimated by detecting and analyzing the changes in locations over a period of time.

Other information such as policy, regulation and priority awareness can be built and stored in the REM database using a kind of machine intelligence and memories. More details about the ways of obtaining various kinds of information in REM can be found in [18].

\footnotetext{
${ }^{2}$ For example, this site (https://www.usgs.gov/) provides geographic and topologic maps in the United Stated.
} 


\subsection{REM applications:}

The IEEE 802.22 standard [23] was the first wireless standard based on cognitive radio technology. It was issued to organize the un-licensed use for Wireless Regional Area Networks (WRANs) operating primarily in the TV broadcast bands (VHF and UHF). In [24] and [25], they expected several potential applications for REMs in WRAN systems. These applications may include network initialization, interference management, efficient spectrum sensing and optimal channel assignment, secondary user transmission power control, fast adaption, positioning enhancement and testing the performance of CRs.

Three major categories of REM application scenarios are introduced in [26]. First scenario is intra-operator radio resource management, which represents cases where networks and frequency bands belong to a single operator. REM can benefit the network operator in many situations. Examples of these situations include performance optimization (interference, handover, etc.), in-band coverage or capacity improvement by relays, and self-configuration and self-optimization of in-band Femto-cells.

The second category of REM application scenarios is the hierarchical spectrum access on licensed bands. This corresponds to the traditional case of dynamic spectrum access where the licensed band is utilized by a secondary user. REM can be useful in coordinated or noncoordinated (spectrum access) between PUs and SUs. Another case for using REM is allowing wireless home network or any local area network operating in TV white space spectrum using CR technology (IEEE 802.11af standard). In addition, REM can also be helpful for LTE coexistence in TV spectrum holes. 
Spectrum sharing on unlicensed bands is the third application scenario. In this category, only several secondary users operate in a spectral environment where there is no primary user. REM can be utilized to fulfill each user requirements and optimize the allocation of resources.

\subsection{Process of designing REM:}

The design of the REM relies on the following related processes: data gathering and representation, and data processing/fusion [21].

\subsubsection{Data gathering and representation:}

Distributed measurement capable devices (MCDs) monitor the environment and report their measurements to the REM manager or fusion center. The measurements may include location of devices, signal levels, time instance and inspected frequency band.

\subsubsection{Data processing/fusion:}

After reporting the measurements to the REM manager, they will be processed to obtain the appropriate physical radio environment model. Based on this model, REM manager can use the measurements to derive the required information to gain a complete awareness of the environment and obtain the REM.

Beside the processing data module in the REM manager, there is also a storage module, which stores the measured and derived data in sort of databases.

Figure 2-3 shows the block diagram that summarizes the designing process of REM, which starts by a request of measurements from the REM manager to the MCDs. In case we already 
know the suitable model, then REM manager can directly generate the REM after getting the measurements.

This generated REM should be updated frequently and consulted each time a CR user wants to launch communication to know the available spectrum and maximum transmitting power that will not cause unacceptable level of interference to a PU.

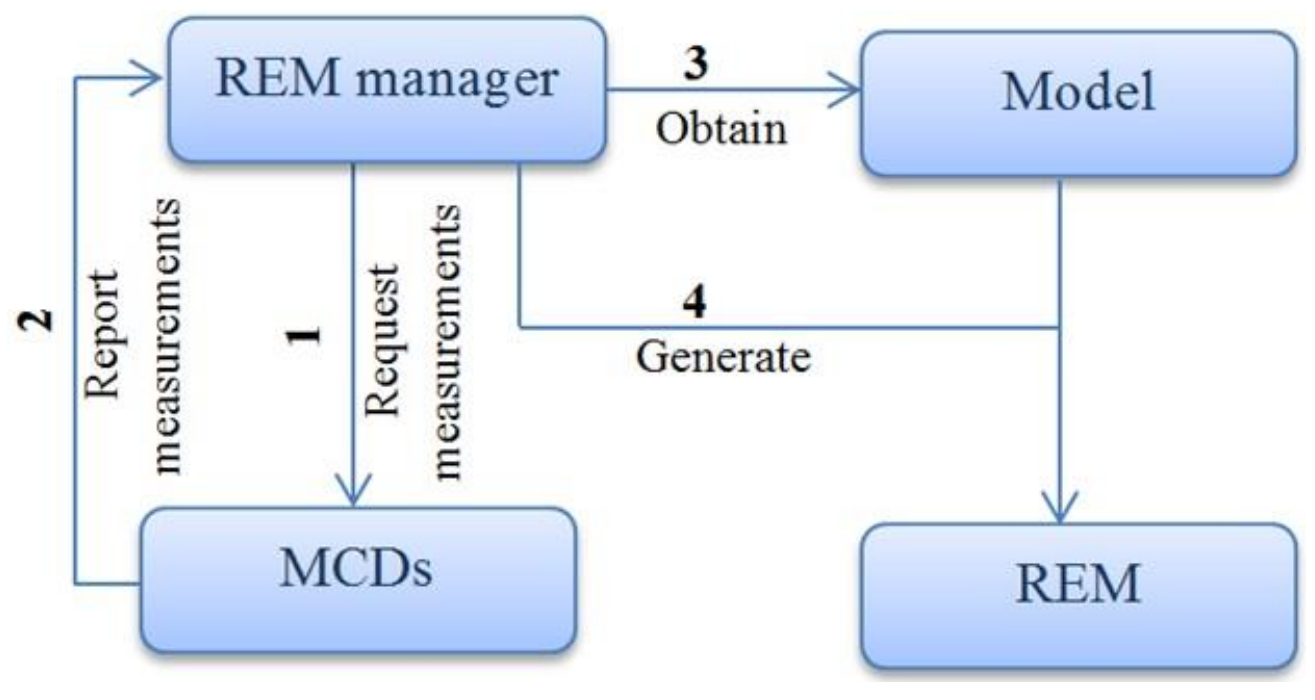

Figure 2-3: REM designing process

\subsection{REM construction Methods:}

REM, as defined earlier, is a complete map that contains different types of information. However, one of the main jobs of a REM is to construct a dynamic interference map for each frequency at each area, and this (radio interference field information) is the most important layer of the REM. Therefore, the focus here is in obtaining a map that displays the estimated level of radio interference over an area of interest, which is also known as "Interference Cartography" (IC). 
Many techniques have been proposed for constructing REM. These techniques can be different from each other in mathematical background, availability of modeling information, or number of primary transmitters in the environment. However, our focus in this thesis is for constructing REM methods with a single active primary user. Thus, in general REM construction methods can be classified in two ways: direct methods and indirect methods [21].

\subsubsection{Direct methods:}

These methods collect some measurements from different points in the area of interest and try to estimate the values of any point in the area. They are based on spatial statistics techniques, which use topological, geometrical or geographical properties of a given area. Processing the measured data to estimate the values of unmeasured points is based on different interpolation techniques.

\subsubsection{Techniques for direct methods:}

There are various kinds of interpolation techniques used for constructing REM or IC. The general principle behind all these spatial interpolation methods is "the closer two together points are in space, the more likely those points are to be similar [27]". Here some of most known interpolation techniques will be briefly presented.

\section{A- Kriging interpolation:}

Kriging is a geostatistical spatial interpolation method that derives predicted values for unmeasured locations by using a weighted average of measured values. Those assigned weights are based on the distance between points in space and the variation between measurements as a function of distance. Kriging process starts by analyzing the spatial variation and then obtaining the predicted values for unmeasured locations. Variograms are used to analyze spatial variation. 
Variogram is a graph that shows the variance of the difference between measurements at different locations as a function of distance between measurements. Kriging method selects weights so that the variance function is minimized. Then, a model is obtained and a curve is fitted to the data in the variogram. Kriging is known as the best linear unbiased estimator, since it minimizes the variance of the difference between measures and estimated values. There are various methods for implementing Kriging interpolation based on the way of calculating the weights. These methods include simple Kriging, ordinary Kriging, universal Kriging, etc. [27].

There has been much research about using this interpolation technique and generation of REM. For example, in [28], distributed Kriging interpolation technique used to obtain interference level map. Also, in [29], they present a probabilistic interference power constraint method for Kriging-based REM. According to [21], this interpolation method requires high number of sensors or MCDs. As a result, high amount of data needs to be processed and the interpolation time will increase.

\section{B- $\quad$ Thin-plate spline interpolation:}

Thin-plate splines were originally introduced in the context of aeroelastic calculations and have been termed surface splines there. Later, mathematicians have laid the theoretical bases of this approach and developed a generalization of it [30]. This interpolation technique has a natural representation in terms of Radial Basis Functions (RBF). It estimates the values for unmeasured locations by using piecewise polynomial functions called splines. The polynomial coefficients are chosen to satisfy smooth passing through all given measured values. Most popular types of splines are linear, quadratic and cubic. In linear spline, the interpolation is formed by straight lines between measured values. Cubic spline obtains interpolated values that are continuous through the second derivative, while quadratic spline concerns about measurements whose first derivative are continuous. 
In [31], they proposed thin-plate spline interpolation to generate interference cartography. They affirm that this interpolation technique neither requires knowledge of spatial statistics, nor relies on stationarity assumptions for generating spectrum cartography.

\section{C- Natural neighbor interpolation:}

This natural-neighbor interpolation method considered in [32], [33], [34] and [35], is based on Voronoi decomposition (tessellation) of a discrete set of spatial points in a plane. The interpolated output values at any point $(x, y)$ can be obtained using a weighted sum of the measured values at its neighbors $\left(x_{i}, y_{i}\right)$

$$
f(x, y)=\sum_{i=1}^{M} w_{i} g\left(x_{i}, y_{i}\right)
$$

Where $M$ is the neighbors of $(x, y), f(x, y)$ is the estimated value at $(x, y), g\left(x_{i}, y_{i}\right)$ are measured known values at $\left(x_{i}, y_{i}\right)$, and $w_{i}$ are the weights, which are calculated as the ratio of the area of overlap to the total area of the new cell in the Voronoi tessellation.

The natural-neighbor interpolation considers only the neighbors in the estimation process, and it requires the interpolated points to be in the convex hull of the measurement locations as the Voronoi cells of outer data points are open-ended polygons with an infinite area. This interpolation method provides a smoother approximation better than simpler methods.

\section{D- Nearest neighbor interpolation:}

This method is also known as polygonal or proximal interpolation. The interpolated predicted values are equal to the nearest measured values, as follow:

$$
f(x, y)=\min _{d_{i}} g\left(x_{i}, y_{i}\right)
$$


where $f(x, y)$ is the predicted value at location $(x, y), g\left(x_{i}, y_{i}\right)$ are measured known values at location $\left(x_{i}, y_{i}\right)$, and $\min _{d_{i}}$ is the minimum Euclidean distance between interpolated and measured points.

This is a very fast and easy interpolation method, but it has less accurate results. It is more suitable for discrete data than continuous one. A further description or implementation of this interpolation method in constructing REM can be found in [27], [35], [33], [36].

\section{E- Inverse distance weighted (IDW):}

This interpolation method [37],[38],[35] is one of the local neighborhood methods, which assume each spatial point influences the spatial field only up to a certain distance by the means of using inverse distance weighting coefficients in the interpolation function. It calculates the interpolated values by using a weighted average sum. The weights are calculated as the inverse of the distance $d_{i}$ between the measured locations and estimated locations and raised to the power $p$, which controls the decrease rate of weight with distance and can be any real number. This method is popular due its ability to operate in different scenarios with limited regular and irregular distributed data. There are different techniques based on inverse distance weighted interpolation [37]: classic IDW method, a modified version of the classical IDW method and a modified Shepard's method.

\subsubsection{Comparative studies of direct methods:}

In the literature, many papers compare between different interpolation techniques in constructing REM. In [34], they compared between three interpolation methods (natural neighbor, thin-plate spline and kriging) for generating interference cartography. The performance and accuracy of each method has been evaluated with different number of sensors and different values of shadowing spread. They concluded that, although those interpolation techniques show 
comparable results, natural neighbor seems to have the best performance among these three techniques.

Another comparative study is provided in reference [39] between three interpolation techniques to generate REM: Kriging, modified Shepard's method (MSM) using IDW and Gradient plus Inverse Distance Squared (GIDS). Their results show that the GIDS interpolation has the lowest performance, whereas Kriging interpolation gives the best performance for constructing REM.

In [33], an evaluation of three interpolation techniques is conducted to acquire situation awareness in cognitive radio. These three techniques are linear interpolation, natural neighbor and nearest neighbor interpolation. The results show that natural neighbor interpolation and linear interpolation perform very similarly and both provide slightly better results than nearest neighbor.

Other comparative studies in various aspects of different interpolation techniques for constructing (IC) or (REM) can be found in [38],[40],[35], [41] and [42].

\subsubsection{Indirect methods:}

The previous presented interpolation techniques obtain interference cartography without requiring transmitter's locations or propagation model to be known. However, the indirect methods try to estimate transmitters' locations and their relevant parameters first, and then by applying the transmitters' estimated parameters in a valid propagation model, we can estimate the signal level at any location in the area of interest. This way of generating REM is a challenging task, because it requires previous knowledge of the propagation model and good approximation of the path loss and other relevant parameters. Based on the availability of known 
information, indirect approaches can be different. Figure 2-4 illustrates the difference between direct and indirect approaches for constructing REM, where the colored areas represent the sensing nodes and different levels of color symbolize different levels of RSS. As seen in the figure, the direct approach consists of two steps: sensing and interpolation, whereas the indirect approach consists of three steps; sensing, estimating transmitter parameters, and utilizing the information of the propagation model to build the REM. 
(A)
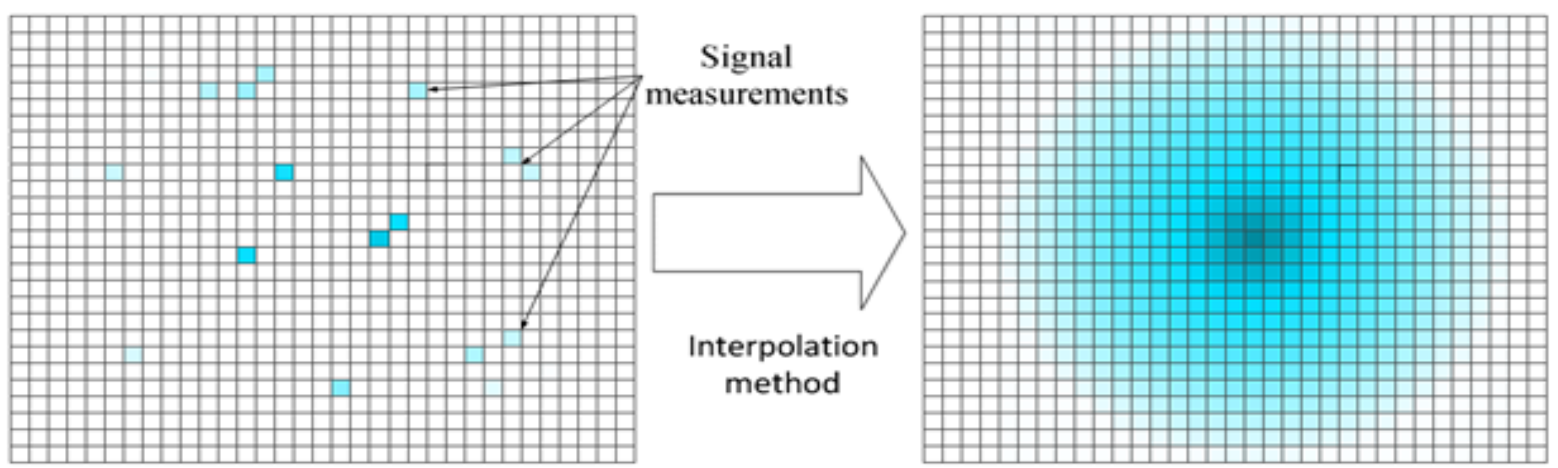

(B)
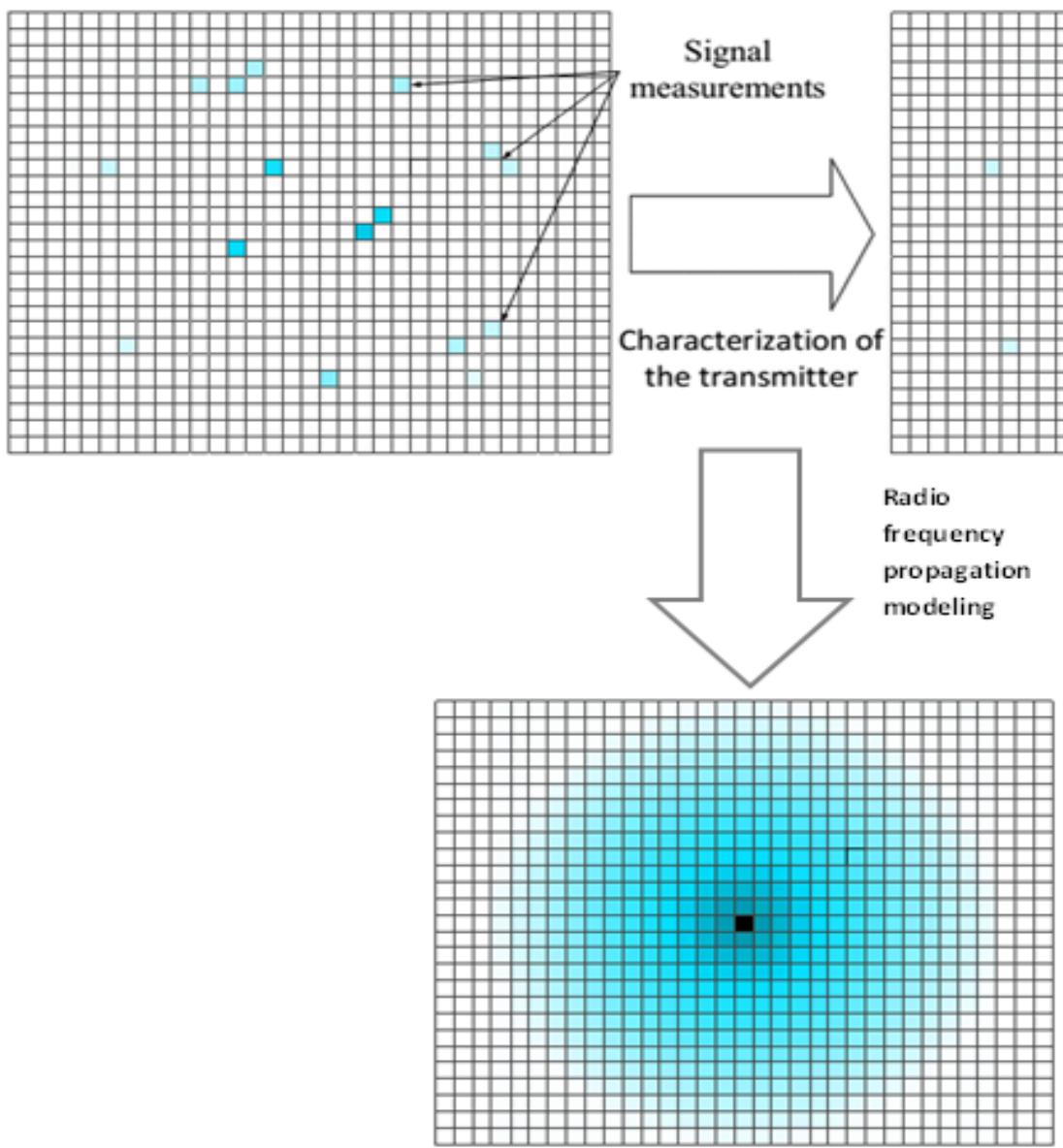

Figure 2-4: The difference between A) direct method and B) indirect method for constructing REM [43] 
Since direct methods are based on localization techniques, an overview of localization parameters will be presented before mentioning some transmitter localization procedures. These parameters can be measured by sensing nodes and reported to the REM manager to locate the transmitter and obtain the REM.

\subsubsection{Localization parameters:}

-Received signal strength (RSS): It is defined as the voltage measured by a sensor or a receiver's received signal strength indicator (RSSI) circuit. Usually, RSS is equivalently reported as measured power. Compared to other parameters, RSS measurements are inexpensive and easy to implement. Shadowing and multipath are two common sources of errors in measured RSS.

-Received signal strength difference (RSSD): This parameter measures the difference in RSS between multiple pair of sensors to locate the transmitter. It is based on this concept that the difference of the received power at two sensors in different locations is related to their distances from the transmitter.

-Time of arrival (TOA): This is the measured time at which the transmitted RF signal first arrives at a receiver. The measured time is composed of transmission time plus a propagationinduced time delay. The core idea of this technique is the ability of the sensing nodes to accurately determine the arrival time of the line-of-sight signal (LOS). There are two major sources that limit the accuracy of the measured time: additive noise and multipath. Localization techniques that use this measured parameter require synchronization between transmitters and sensing nodes.

-Angle of arrival (AOA): Positioning techniques using this parameter are based on measuring angles of the transmitted signals by sensing nodes. There are two common ways for sensing nodes to measure AOA: a) from the differences in arrival times for a transmitted 
signal at each of the sensor array elements, or b) by using the RSS ratio between two (or more) directional antennas located on the sensors. As in TOA measurements, additive noise and multipath are the main sources that affect the measured values.

Further details about localization parameters and techniques that use these parameters, and the advantages and disadvantages of each technique can be found in [44],[45],[46]. However, because RSS values are the simplest to measure and there is no need for synchronization between primary and secondary networks, our interest here are for localization methods based on RSS or RSSD, which can be used to construct REMs.

\subsubsection{Techniques for indirect methods:}

As explained previously, the indirect methods utilize the known or estimated propagation model information to locate the transmitter and then construct the REM. Based on the amount of known parameters, and the mathematical approach, localization techniques can be different. Here, some methods for estimating transmitter location in different cases will be mentioned briefly.

In [47], [48], they presented an iterative grid search method for locating the primary transmitter in CRNs based on RSS by secondary networks. They assume only the path loss exponent of the log-distance model is known. However, to use this method for constructing REM, a way of estimating the transmitted power is required to obtain the interference level at any location in the environment.

In [49], they used Metropolis-Hastings $(\mathrm{MH})$ algorithm to locate the primary emitter and generate the REM. In this method, they assume the transmitted power and model parameters are known except shadowing spread value. However, to reduce the complexity of the (MH) algorithm, it can be integrated with a low complexity interpolation technique. 
In [50], four least square methods based on received signal strength difference (RSSD) are evaluated in terms of correctly finding transmitter location. However, these methods in general can't be used directly for obtaining REM, because they only estimate the transmitter location without its transmitted power. But if the transmitted power is pre-known, these methods can be utilized to obtain a REM.

Another way based on (RSS) is proposed in [51] to estimate the transmitter location and its transmitted power and then utilize them to construct REM. They also compare its performance with other direct interpolation methods (Kriging and inverse distance weighted interpolation). Their comparison results show that this indirect method outperforms aforementioned spatial interpolation techniques.

\subsubsection{Comparative studies of indirect methods:}

This topic of indirect ways to construct REM based on estimation of transmitter parameters is still a new field in REM design. In comparison to direct interpolation techniques, there is not much research about this indirect ways for constructing REM. Consequently, not much of comparative studies between indirect methods are presented in the literature. However, a summarized survey of some direct and indirect methods for constructing REM, and pros and cons of each method is presented in [35]. In [42], a performance comparison between some direct methods and two indirect methods is introduced.

Studying the performance of direct methods requires inspecting several factors, e.g. number of sensors, accuracy of modeling, mobility, etc. Therefore, further comparisons and 
investigation in different scenarios for some indirect methods will be presented in the next chapters.

\subsection{REM quality metrics:}

After processing the measurements collected by the MCDs distributed in the area, estimated interference cartography, which represents the most important part of REM, can be generated. There are several parameters that affect the performance of the REM such as number of sensors, their distribution in the area, accuracy of sensing, movement of sensors and accuracy of the propagation model. Therefore, the accuracy of the estimated REM is evaluated through several metrics in terms of some of the aforementioned factors. These metrics are:

\subsubsection{Localization error:}

This is defined as the difference in meters between the estimated and actual transmitter locations.

\subsubsection{Signal power error:}

This is calculated as the difference in $\mathrm{dB}$ between the actual and estimated transmitter power. For this quality metric and the previous one, the difference can be calculated as the absolute value, or can be expressed using the Euclidean metric, which gives the average Root Mean Squared Error (RMSE). In our simulations, we will use the absolute value metric.

\subsubsection{Correct Detection Zone Ratio and False Alarm Zone Ratio:}

The previous quality metrics describe the goodness of the estimated REM, but they can't identify the regions where the spectrum opportunities are lost. Therefore, the two metrics Correct Detection Zone Ratio (CDZR) and False Alarm Zone Ratio (FAZR) are presented in [51]. 
A contour around the active transmitter is constructed to represent the prohibited area of transmission for the secondary user. The radius of the constructed contour is obtained based on the minimum detectable signal of the primary user, which is set by the regulators.

The error in estimating the transmitter parameters results in errors for the estimated REM contour, as depicted in Figure 2-5. The intersected area between the actual and estimated REM contours $\left(\mathbf{Z}_{\mathbf{1}}{ }^{\mathbf{C D}}\right)$ represents the correct detection zone for the active transmitter, and the prohibited area for the secondary user. Likewise, $\left(\mathbf{Z}_{\mathbf{0}}{ }^{\mathbf{C D}}\right)$ defines the correct detection for free transmission zones. In contrast, the area where the transmission is estimated to be forbidden but it is actually allowed denoted as False Alarm Zone $\left(\mathbf{Z}^{\mathbf{F A}}\right)$. In a similar manner, missed detection zone $\left(\mathbf{Z}^{\mathrm{MD}}\right)$ refers to an area where transmission is forbidden, but estimated to be allowed because of estimation errors. The Correct Detection Zone Ratio (CDZR) and False Alarm Zone Ratio (FAZR) defined as follow:

$$
\begin{aligned}
& F A Z R=\frac{A\left(Z^{F A}\right)}{A\left(Z^{F A}\right)+A\left(Z_{0}^{C D}\right)} \\
& C D Z R=\frac{A\left(Z_{1}^{C D}\right)}{A\left(Z_{1}^{C D}\right)+A\left(Z^{M D}\right)}
\end{aligned}
$$

where $A(Z)$ represents the area of the zone.

These two metrics define correctly the area where the primary user is harmed and the area where the spectrum opportunity is lost. Having higher (CDZR) indicates good fit of the estimated REM with the true REM and less probability of harming the primary user. On the other hand, having higher FAZR means bad utilization of the spectrum opportunities. These 
metrics are also analogous to the probability of detection and false alarm in the spectrum sensing context.

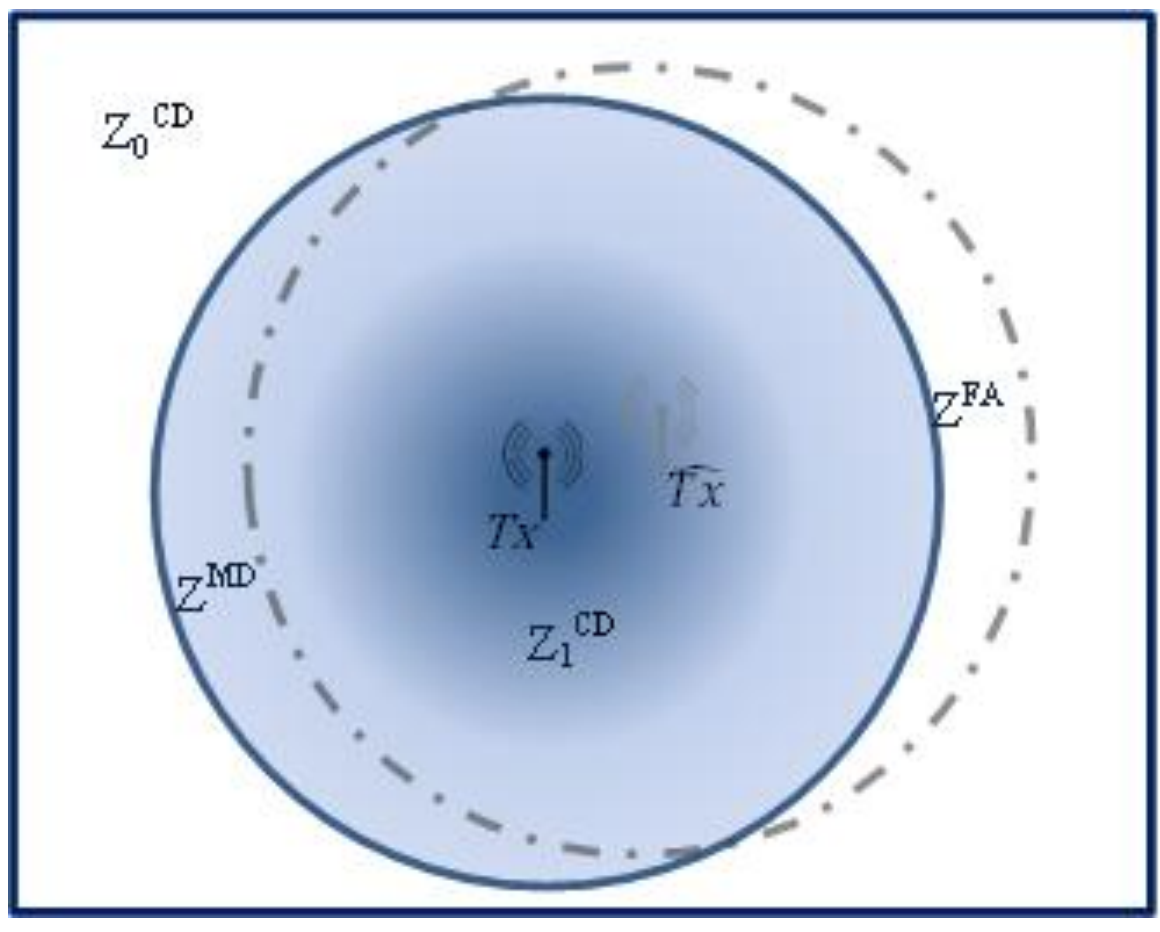

Figure 2-5: Actual and estimated REM contours 


\section{Chapter 3: System Models}

In the previous chapter, we described the necessity of comparing between some indirect methods for constructing REM and testing them in different scenarios. Here, the mathematical analyses of the methods are presented, and in the next chapter the simulation results are provided. The chapter starts by presenting the mathematical background of the log-normal shadowing model. Then, two indirect methods based on that model to estimate transmitter parameters and construct REM will be explained in details. The comparison between them will be further explored in the next chapter.

\subsection{Signal power and distance:}

Practical experiments have shown that, average received power in radio channels decrease logarithmically with distance. Therefore, average path-loss is expressed as a function of distance [52]:

$$
\overline{P L}(d) \propto\left(\frac{d}{d_{0}}\right)^{\alpha}
$$

or

$$
\overline{P L}(d B)=\overline{P L}\left(d_{0}\right)+10 \alpha \log _{10}\left(\frac{d}{d_{0}}\right)
$$

where $\alpha$ is the path-loss exponent representing the rate of path-loss increment with distance and it is dependent on the propagation environment, $d$ is the distance from the transmitter to the receiver, and $d_{0}$ is the reference distance which is selected in accordance with propagation 
environment. For example, $1 \mathrm{~km}$ is used as reference distances in macrocell systems, while much smaller distances (such as $100 \mathrm{~m}$ or $1 \mathrm{~m}$ ) are used in microcell systems [21]. The reference pathloss $\overline{P L}\left(d_{0}\right)$ is obtained through field measurements at distance $d_{0}$ or can be calculated using this formula [52]:

$$
P L(d B)=-10 \log \left[\frac{G_{t} G_{r} \lambda^{2}}{(4 \pi)^{2} d^{2}}\right]
$$

Where $G_{t}$ is the transmitter antenna gain, $G_{r}$ is the receiver antenna gain, $d$ is the separation distance between the transmitter and the receiver, and $\lambda$ is the wavelength in meters.

\subsection{Signal power under log-normal shadowing:}

The model in Eq.(3-2) neglects the fact that the measured value of RSS at different locations within the same separation distance may be immensely different. This is due to shadowing effect which is caused by obstacles such as hills or large buildings in the propagation path between the transmitter and the receiver. The change in RSS is often modeled using a lognormal distribution (normal in $\mathrm{dB}$ ). Therefore, Eq.(3-2) can be rewritten as [52]:

$$
P L(d)[d B]=\overline{P L}(d)+X(0, \sigma)=\overline{P L}\left(d_{0}\right)+10 \alpha \log _{10}\left(\frac{d}{d_{0}}\right)+X(0, \sigma)
$$

where $X(0, \sigma)$ is a zero-mean Gaussian distributed random variable (in $\mathrm{dB}$ ) with standard deviation $\sigma$ (also in $\mathrm{dB}$ ). Thus, the relation between the transmitted signal and received signal is:

$$
P_{r}(d)[d B m]=P_{t}[d B m]-P L(d)[d B]
$$

Eq.(3-4) implies that measured signal levels (in $\mathrm{dB}$ ) at specific distances from the transmitter have Gaussian (normal) distribution about the distance-dependent mean of Eq.(3-2). 


\subsection{Mathematical description of some indirect methods to construct REM:}

The first step of the indirect ways to obtain REM is to estimate the transmitter parameters. Here, two methods to estimate transmitter parameters will be presented. The first one is based on received signal strength difference (RSSD), and the second one is based on received signal strength (RSS).

\subsubsection{RSSD method:}

If we consider an environment where all the transmitters in the area have similar known transmitted power, then we only need to find the transmitter location to generate REM. In [50], they presented four geolocation techniques based on RSSD to locate the emitter. The basic idea behind these techniques is that the ratio of the signal powers (or their differences expressed in $\mathrm{dB}$ ) observed at two different sensing locations is related to the ratios of the emitter to sensor distances.

The received signal power is given by:

$$
P_{r}(d)=P\left(d_{0}\right)-10 \alpha \log _{10}\left(\frac{d}{d_{0}}\right)
$$

For two sensors located at $\left(x_{1}, y_{1}\right)$ and $\left(x_{2}, y_{2}\right)$, the measured received signal at each sensor is given by:

$$
\begin{aligned}
& P_{1}\left(d_{1}\right)=P\left(d_{0}\right)-10 \alpha \log _{10}\left(\frac{d_{1}}{d_{0}}\right) \\
& P_{2}\left(d_{2}\right)=P\left(d_{0}\right)-10 \alpha \log _{10}\left(\frac{d_{2}}{d_{0}}\right)
\end{aligned}
$$

Then, the power difference between these two sensors is: 


$$
\begin{gathered}
\boldsymbol{P}_{12}=P_{1}\left(d_{1}\right)-P_{2}\left(d_{2}\right)=10 \alpha\left[\log _{10}\left(d_{0}\right)-\log _{10}\left(d_{1}\right)+\log _{10}\left(d_{2}\right)-\right. \\
\left.\log _{10}\left(d_{0}\right)\right]=\mathbf{1 0} \alpha \log _{10}\left(\frac{\boldsymbol{d}_{2}}{\boldsymbol{d}_{\mathbf{1}}}\right)
\end{gathered}
$$

The distance between the primary user transmitter and any sensor located at $\left(x_{k}, y_{k}\right)$ is obtained as:

$$
d_{k}=\sqrt{\left(x_{p u}-x_{k}\right)^{2}+\left(y_{p u}-y_{k}\right)^{2}}
$$

Using both Eq.(3-10) along with Eq.(3-9) yields:

$$
P_{12}=10 \alpha \log _{10}\left(\frac{\left(x_{p u}-x_{2}\right)^{2}+\left(y_{p u}-y_{2}\right)^{2}}{\left(x_{p u}-x_{1}\right)^{2}+\left(y_{p u}-y_{1}\right)^{2}}\right)^{1 / 2}
$$

In general, for any two sensors located at $\left(x_{k}, y_{k}\right)$ and $\left(x_{m}, y_{m}\right)$ :

$$
P_{k m}=P_{k}-P_{m}=5 \alpha \log _{10}\left(\frac{\left(x_{p u}-x_{m}\right)^{2}+\left(y_{p u}-y_{m}\right)^{2}}{\left(x_{p u}-x_{k}\right)^{2}+\left(y_{p u}-y_{k}\right)^{2}}\right)
$$

To solve the previous equation to obtain the transmitter location, four approaches are presented and compared in [50], three linear approaches and one non-linear approach. The best performance of linear methods is introduced here.

Let: $\beta_{k m}=\frac{\left(x_{p u}-x_{m}\right)^{2}+\left(y_{p u}-y_{m}\right)^{2}}{\left(x_{p u}-x_{k}\right)^{2}+\left(y_{p u}-y_{k}\right)^{2}}$, then by substituting in Eq.(2-2):

$$
\beta_{k m}=\frac{\left(x_{p u}-x_{m}\right)^{2}+\left(y_{p u}-y_{m}\right)^{2}}{\left(x_{p u}-x_{k}\right)^{2}+\left(y_{p u}-y_{k}\right)^{2}}=10^{\frac{P_{k m}}{5 \alpha}}
$$

Hence,

$$
\left(x_{p u}-x_{m}\right)^{2}+\left(y_{p u}-y_{m}\right)^{2}=\beta_{k m}\left[\left(x_{p u}-x_{k}\right)^{2}+\left(y_{p u}-y_{k}\right)^{2}\right]
$$

After analyzing the squared expression and re-arrangement, we get: 


$$
\begin{gathered}
\left(1-\beta_{k m}\right)\left(x_{p u}^{2}+y_{p u}^{2}\right)-2\left(x_{m}-\beta_{k m} x_{k}\right) x_{p u}-2\left(y_{m}-\beta_{k m} y_{k}\right) y_{p u} \\
=\beta_{k m}\left(x_{k}^{2}+y_{k}^{2}\right)-\left(x_{m}^{2}+y_{m}^{2}\right)
\end{gathered}
$$

or,

$$
\left(1-\beta_{k m}\right) C-2\left(x_{m}-\beta_{k m} x_{k}\right) x_{p u}-2\left(y_{m}-\beta_{k m} y_{k}\right) y_{p u}=Z_{k m}
$$

where

$$
C=x_{p u}^{2}+y_{p u}^{2}, Z_{k m}=\beta_{k m}\left(x_{k}^{2}+y_{k}^{2}\right)-\left(x_{m}^{2}+y_{m}^{2}\right)
$$

If we define a matrix $\mathbf{A}$ with size $(N-1) \times 3$ consisting of the row vectors, [( $1-$ $\left.\left.\beta_{1 k}\right),-2\left(x_{k}-\beta_{1 k} x_{1}\right),-2\left(y_{k}-\beta_{1 k} y_{1}\right)\right], 2 \leq k \leq N$, and vector b with size $(N-1)$ whose elements are the terms of $Z_{1 k}, 2 \leq k \leq N$, then Eq.(2-2) can be written in the matrix form $A X=b$ as follows:

$$
\left[\begin{array}{ccc}
1-\beta_{12} & -2\left(x_{2}-\beta_{12} x_{1}\right) & -2\left(y_{2}-\beta_{12} y_{1}\right) \\
1-\beta_{13} & -2\left(x_{3}-\beta_{13} x_{1}\right) & -2\left(y_{3}-\beta_{13} y_{1}\right) \\
\vdots & \vdots & \vdots \\
1-\beta_{1 N} & -2\left(x_{N}-\beta_{1 N} x_{1}\right) & -2\left(y_{N}-\beta_{1 N} y_{1}\right)
\end{array}\right]\left[\begin{array}{c}
C \\
x_{p u} \\
y_{p u}
\end{array}\right]=\left[\begin{array}{c}
Z_{12} \\
Z_{13} \\
\vdots \\
Z_{1 N}
\end{array}\right]
$$

This is a linear system which we can estimate its solution as follow:

$$
\left[\widehat{C}, \widehat{x_{p u}}, \widehat{y_{p u}}\right]^{T}=\left(A^{T} A\right)^{-1} A^{T} \mathbf{b}
$$

By estimating the location of the primary user transmitter, we can estimate the received signal level at any location in the area by utilizing Eq. (3-5). Hence, REM can be generated.

\subsubsection{RSS method:}

Combining analytical models with a statistical evaluation through measurements, a practical model for the RSS at location $i$ is proposed in [21] :

$$
P_{r i}(d)[d B m]=P_{t}[d B m]-l_{0}-10 \alpha \log _{10}\left(d_{i}[m]\right)+X(0, \sigma)
$$

where $P_{t}$ is the transmitted power and $l_{0}$ is the path-loss constant. 
The values of path-loss constant $\left(l_{0}\right)$, path-loss exponent $(\alpha)$, and standard deviation $(\sigma)$ depend on the environment and propagation scenario. They are experimentally computed from measured data, using linear regression such that the difference between the measured and estimated path losses is minimized in mean square error sense over a wide range of measurement locations and different separation distances from the transmitter [52]. Table 1 lists typical path loss exponents obtained in various mobile radio environments, and Table 2 lists values for pathloss constant $\left(l_{0}\right)$ and shadow fading standard deviation $(\sigma)$ for some different scenarios.

Table 1: Path-loss Exponents for Different Environments [52]

\begin{tabular}{|lc|}
\hline Environment & Path-loss exponent $(\boldsymbol{\alpha})$ \\
\hline Free space & 2 \\
\hline Urban area cellular radio & 2.7 to 3.5 \\
\hline Shadowed urban cellular radio & 3 to 5 \\
\hline In building (LOS) & 1.6 to 1.8 \\
\hline Obstructed in building & 4 to 6 \\
\hline Obstructed in factories & 2 to 3 \\
\hline
\end{tabular}


Table 2: path-loss constant and standard deviation in different scenarios [53]

\begin{tabular}{|c|c|c|c|c|}
\hline Scenario & $\begin{array}{l}\text { LOS / } \\
\text { NLOS }\end{array}$ & $\begin{array}{c}\text { Path-loss } \\
\text { constant }\left(l_{0}\right) \\
{[\mathrm{dB}]}\end{array}$ & $\begin{array}{c}\text { standard } \\
\text { deviation }(\sigma) \\
{[\mathrm{dB}]}\end{array}$ & $\begin{array}{c}\text { applicability } \\
\text { range }\end{array}$ \\
\hline \multirow{2}{*}{ Indoor small office } & LOS & 46.8 & 3.1 & $3 \mathrm{~m}<\mathrm{d}<100 \mathrm{~m}$ \\
\hline & NLOS & 38.8 & 3.5 & $3 \mathrm{~m}<\mathrm{d}<100 \mathrm{~m}$ \\
\hline Urban micro-cell & LOS & 41 & 2.3 & $10 \mathrm{~m}<\mathrm{d}<650 \mathrm{~m}$ \\
\hline Indoor hotspot & LOS & 36.9 & 1.4 & $5 \mathrm{~m}<\mathrm{d}<29 \mathrm{~m}$ \\
\hline Suburban macro-cell & NLOS & 27.7 & 8 & $50 \mathrm{~m}<\mathrm{d}<5 \mathrm{~km}$ \\
\hline Urban macro-cell & NLOS & 38.4 & 8 & $50 \mathrm{~m}<\mathrm{d}<5 \mathrm{~km}$ \\
\hline
\end{tabular}

In this method and in the previous method, we assume that we can determine the values of the propagation model parameters $\left(\alpha, \sigma\right.$ and $l_{0}$ 'for second method") based on the tables in the literature. We just need to estimate the transmitter parameters (location and emitted power) to estimate the power level at any location in the area of interest. In the RSSD method, we also assumed that all transmitters in the area have pre-known transmitted power. However, using Eq.(3-19), an approach in [51] is suggested to estimate the transmitter location and its transmitted power.

The measured values of RSS by sensors under log-normal shadowing channel subject to random fluctuations. Therefore, to reduce the shadowing effect, RSS measured $N$ times and the average value is obtained as

$$
\overline{P_{r l}}(d)=\sum_{j=1}^{N} \frac{P_{r i j}(d)}{N}
$$


where $P_{r i j}(d)$ is the $\mathrm{j}^{\text {th }}$ received power at $\mathrm{i}^{\text {th }}$ sensor and $N$ is the number of measurements in the sample.

If the sample is large enough, the average received power at $i^{\text {th }}$ sensor can be approximated as

$$
\overline{P_{r l}}(d) \approx P_{t}-l_{0}-10 \alpha \log _{10}\left(d_{i}\right)
$$

After rearranging and taking the log of both sides

$$
\left(d_{i}\right) \approx 10^{\frac{P_{t}-l_{0}-\overline{P_{r l}}(d)}{10 \alpha}}
$$

Since $\left(d_{i}\right)$ represents the distance between the transmitter and $\mathrm{i}^{\text {th }}$ sensor, we can write

$$
\sqrt{\left(x_{t}-x_{i}\right)^{2}+\left(y_{t}-y_{i}\right)^{2}} \approx 10^{\frac{P_{t}-l_{0}-\overline{P_{r l}}(d)}{10 \alpha}}
$$

By squaring both sides

$$
x_{t}^{2}-2 x_{i} x_{t}+x_{i}^{2}+y_{t}^{2}-2 y_{i} y_{t}+y_{i}^{2} \approx 10^{\frac{P_{t}-l_{0}-\overline{P_{l}}(d)}{5 \alpha}}
$$

This can be rearranged and transformed to a regression problem as follows:

$$
10^{\frac{-l_{0}-\overline{P_{r l}}(d)}{5 \alpha}} 10^{\frac{P_{t}}{5 \alpha}}+2 x_{i} x_{t}+2 y_{i} y_{t}-C \approx x_{i}{ }^{2}+y_{i}{ }^{2}
$$

where $C=x_{t}^{2}+y_{t}^{2}$

Hence, Eq.(2-2) can be expressed in a matrix form $A X=b$

where

$$
A=\left[\begin{array}{cccc}
10^{\frac{-l_{0}-\overline{P_{r 1}}(d)}{5 \alpha}} & 2 x_{1} & 2 y_{1} & -1 \\
10^{\frac{-l_{0}-\overline{P_{r 2}}(d)}{5 \alpha}} & 2 x_{2} & 2 y_{2} & -1 \\
\vdots & \vdots & \vdots & \vdots \\
10^{\frac{-l_{0}-\overline{P_{r n}}(d)}{5 \alpha}} & 2 x_{n} & 2 y_{n} & -1
\end{array}\right], X=\left[\begin{array}{c}
10 \frac{P_{t}}{5 \alpha} \\
x_{t} \\
y_{t} \\
C
\end{array}\right] \text { and } b=\left[\begin{array}{c}
x_{1}{ }^{2}+y_{1}{ }^{2} \\
x_{2}{ }^{2}+y_{2}{ }^{2} \\
\vdots \\
x_{n}{ }^{2}+y_{n}{ }^{2}
\end{array}\right]
$$


This matrix representation can be solved using least-square methods, which gives minimum achievable error. Hence, we get estimated values for the transmitted power $\widehat{P}_{t}$ and the location of the transmitter $\left(\widehat{x_{t}}, \widehat{y_{t}}\right)$. Using these estimated data, we can construct the REM by evaluating the estimated received power at any location, and this is done by first calculating the distance from the estimated location to the transmitter as follows:

$$
\begin{gathered}
\hat{d}=\sqrt{\left(\widehat{x_{t}}-x\right)^{2}+\left(\widehat{y_{t}}-y\right)^{2}} \\
\widehat{P_{r}}(d)=\widehat{P}_{t}-l_{0}-10 \alpha \log _{10}(\hat{d})
\end{gathered}
$$

Comparison between these two methods and simulation results is presented in the next chapter. 


\section{Chapter 4: Simulation and Results}

This chapter compares between the two indirect methods for constructing REM explained in the preceding chapter. The method that shows better performance will undergo several performance tests in different scenarios and conditions. These scenarios include different number of sensors, varied values of shadowing spread, diverse sample size of measurements, sensitivity to path loss exponent, multiple moving with fixed or different speeds.

In all the scenarios, we consider an environment where there is only one active transmitter in the area of interest, and therefore a single channel is available at a certain time.

\subsection{Comparison between RSSD and RSS methods:}

\subsubsection{Simulation description:}

To test the performance of the REM constructed by the two algorithms described previously, the localization error metric of transmitter is considered. The considered environment is an urban macro-cell square area of $1 \mathrm{~km}^{2}$. Accordingly, the parameters of path- loss exponent $(\alpha)$, path-loss constant $\left(l_{0}\right)$ and shadow fading standard deviation $(\sigma)$ have been selected as follow: $\alpha=3.5, l_{0}=38.4$ and $\sigma=8$ (See Table 1 and Table 2). Because we consider a homogeneous symmetric environment, all the aforementioned parameters are assumed to have fixed constant values. The transmitted power is set to $(27 \mathrm{dBm})$ and the location of the transmitter is selected randomly. Also, twenty sensors are distributed randomly in the area. In order to improve the relevance of the results and to validate the model in an urban area as required in Table 2, a minimum distance of $50 \mathrm{~m}$ between the sensors and between the 
transmitter and any sensor is considered in the simulation, as shown in (Figure 4-1). Random zero-mean Gaussian noise with a standard deviation $(\sigma)$ of $8 \mathrm{~dB}$ is added to the path-loss expression to represent the shadowing effect. The experiment is conducted by using MATLAB and the average received RSS by each sensor is calculated using Eq.(3-5) with the previous considered parameters.

\subsubsection{Simulation results:}

The two algorithms for estimating the transmitter location are implemented as described in the previous chapter. The performance is tested with different numbers of distributed sensors as shown in Figure 4-2. The minimum number of sensors to apply RSSD algorithm is three sensors, whereas for RSS algorithm it is four sensors, as illustrated in Eq.(3-17) and the matrix form of Eq.(3-25). However, since the simulation results give unstable results for less than five distributed sensors, and to get a fair comparison between the two algorithms, the minimum number of distributed sensors is limited to six sensors.

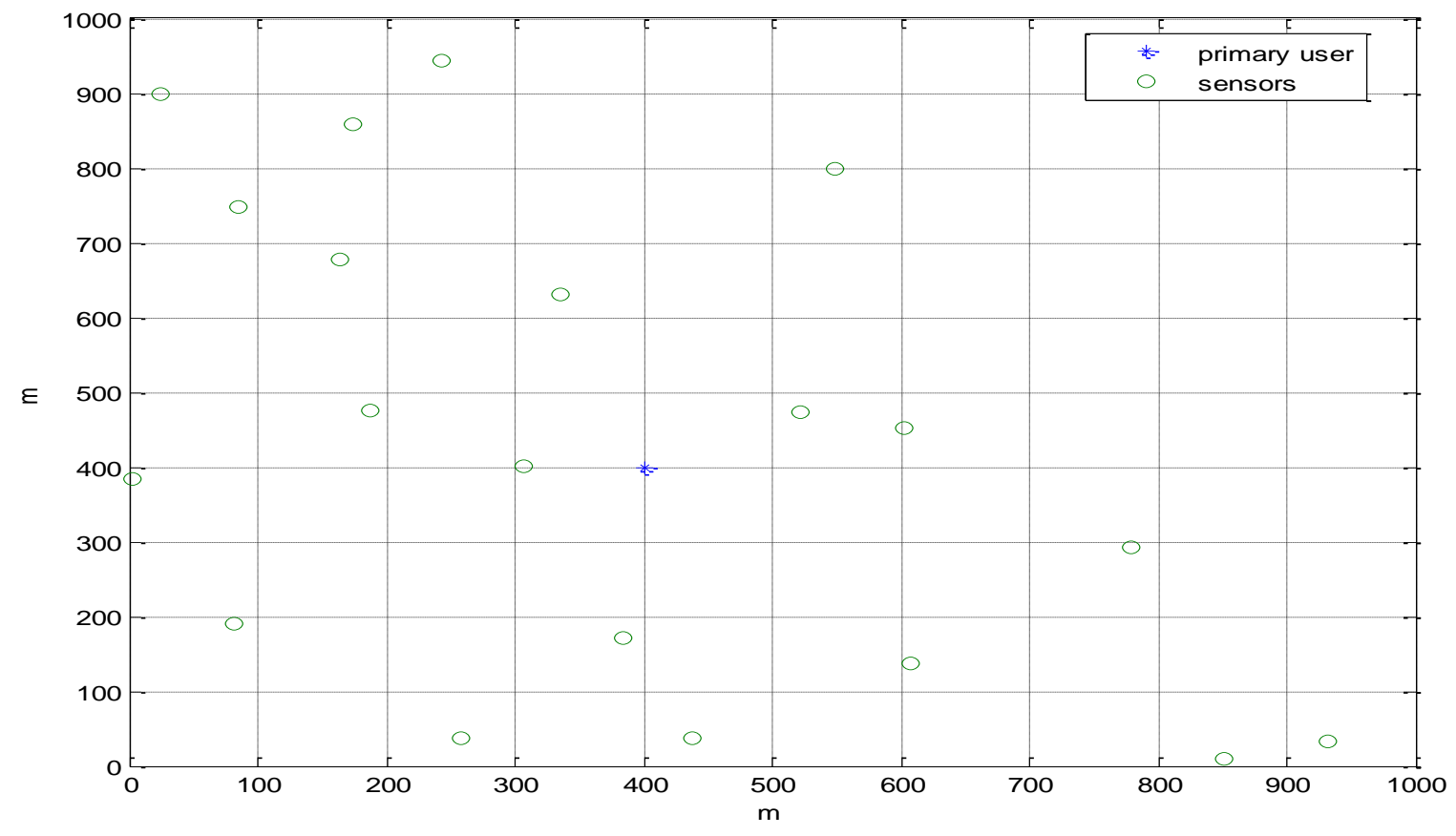

Figure 4-1: Distribution of sensors around the primary user transmitter 


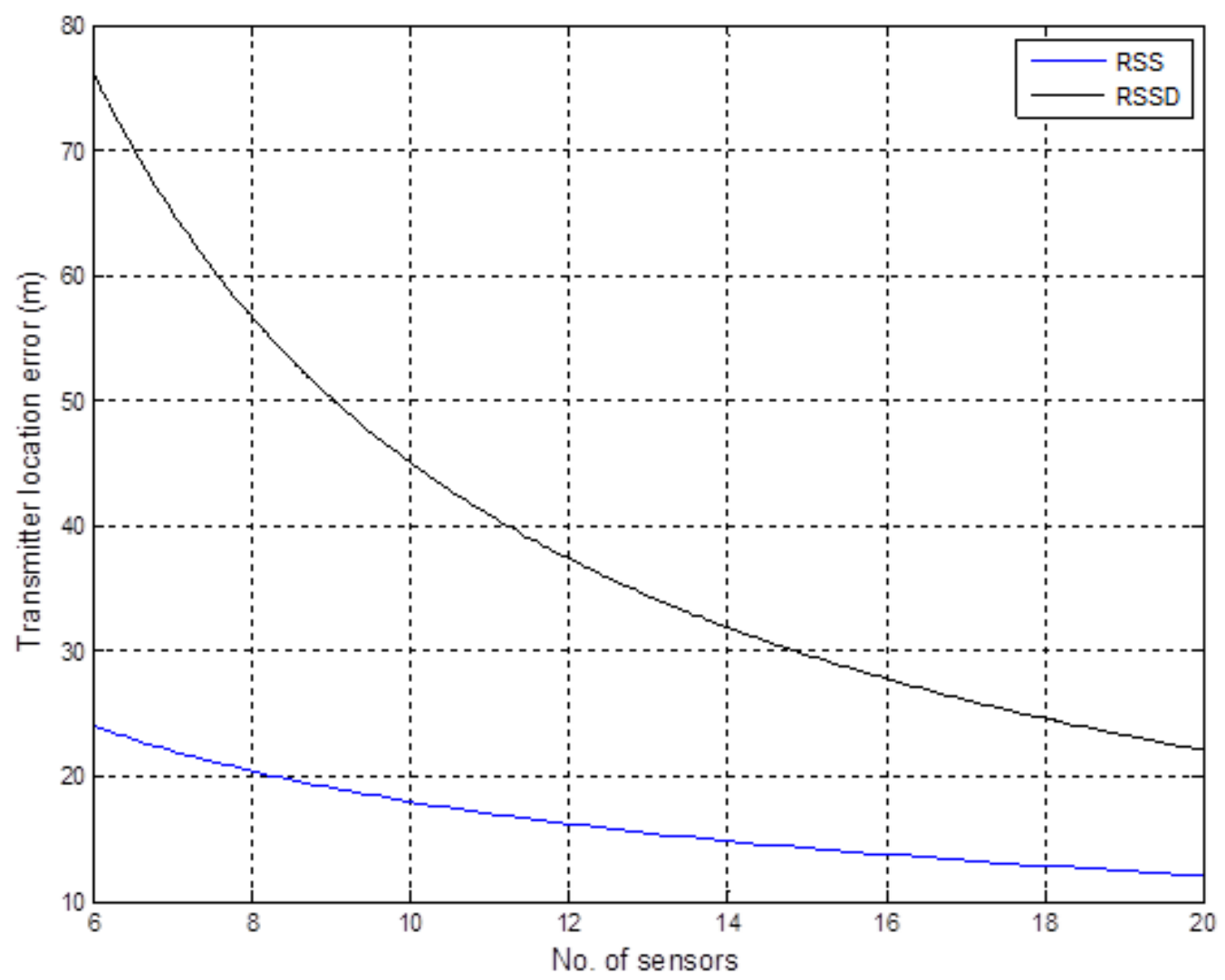

Figure 4-2: Performance comparison between RSS and RSSD algorithms

$$
\left(\alpha=3.5, l_{0}=38.4 \text { and } \sigma=8\right)
$$

From the simulation result, we see that in both algorithms increasing the number of distributed sensors in the area will increase the accuracy of estimation. However, it is clear that the algorithm used for RSS gives much better performance than the algorithm applied using RSSD. Also, the estimation of the localization error becomes stable faster in RSS algorithm than in RSSD algorithm. In the RSSD method, we did not use the reference path-loss in the estimation process, whereas RSS method assumes path-loss constant, which is similar to reference pathloss, can be known and utilized in the estimation process. 
In comparison between the obtained results and previous work, RSS method was not examined with localization metric in the literature, whereas the performance of RSSD method was tested in [50] and the results show a large range of errors in comparison with our simulated results. They got an average error of the transmitter location ranges from $1000 \mathrm{~m}$ to $600 \mathrm{~m}$ as the number of sensors is changing from 5 to 15 sensors, while in our results the error changes approximately from $80 \mathrm{~m}$ to $25 \mathrm{~m}$ as the number of sensors increases from 6 to 20 sensors. The reason for this big difference in localization errors is because they obtained the RSS values experimentally by using a mobile data collection vehicle. The vehicle moves in the area of interest with a fixed speed to measure RSS values along with the GPS coordinates. They considered an area of interest of $9 \mathrm{~km}^{2}(3 \mathrm{~km} \times 3 \mathrm{~km})$, and the measured results are subjected to log-normal model with a path-loss exponent of 3.75. The other parameters (transmitter power and standard deviation) are set as in our simulation. One the other hand, our simulation calculates the RSS values for known locations of sensors that are distributed in a square area of 1 $\mathrm{km}^{2}$. In both experiments, the path-loss exponent is assumed as a fixed value for all location, but practically it may change from place to another. Based on all aforementioned factors, we can understand the reasons of the differences in the localization error between the two experiments.

Based on the results shown in Figure 4-2, it can be stated that RSS algorithm is more accurate and reliable than RSSD algorithm. However, RSS algorithm is preferred if we have a good estimation of path-loss constant value. Therefore, RSS method is selected to be further investigated in different scenarios. 


\subsection{Simulation Scenarios:}

\subsubsection{Scenario 1: (performance test with different number of sensors)}

The environment considered for this scenario is the same as the previous simulation. The performance will be tested with the other quality metrics (Signal power error and CDZR).

The error of the estimated transmitter power versus the number of distributed sensors is shown in Figure 4-3. To construct the contour that represents the prohibited zone of transmission for the secondary user around the primary user, the level of $\mathbf{- 1 1 4} \mathbf{~} \mathbf{~ B m}$ is considered as the minimum detectable signal level of the primary user, as it is required by FCC regulations [20]. The correct detection zone ratio (CDZR), which represents the percentage of the correct decision of detecting the primary user, is plotted versus different number of the distributed sensors, as shown in Figure 4-4. 


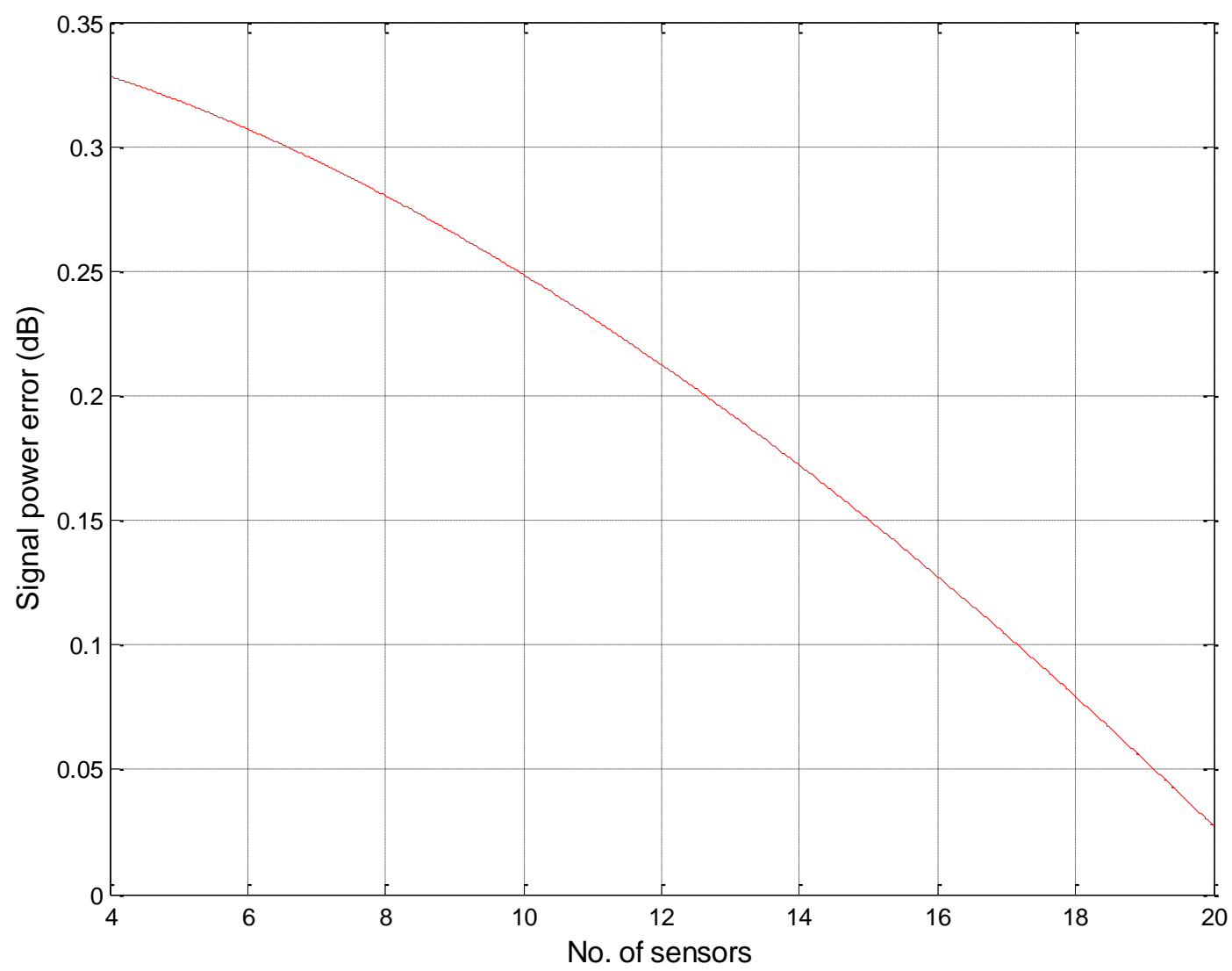

Figure 4-3: Number of sensors versus error of the estimated transmitted power

$$
\left(\alpha=3.5, l_{0}=38.4 \text { and } \sigma=8\right)
$$




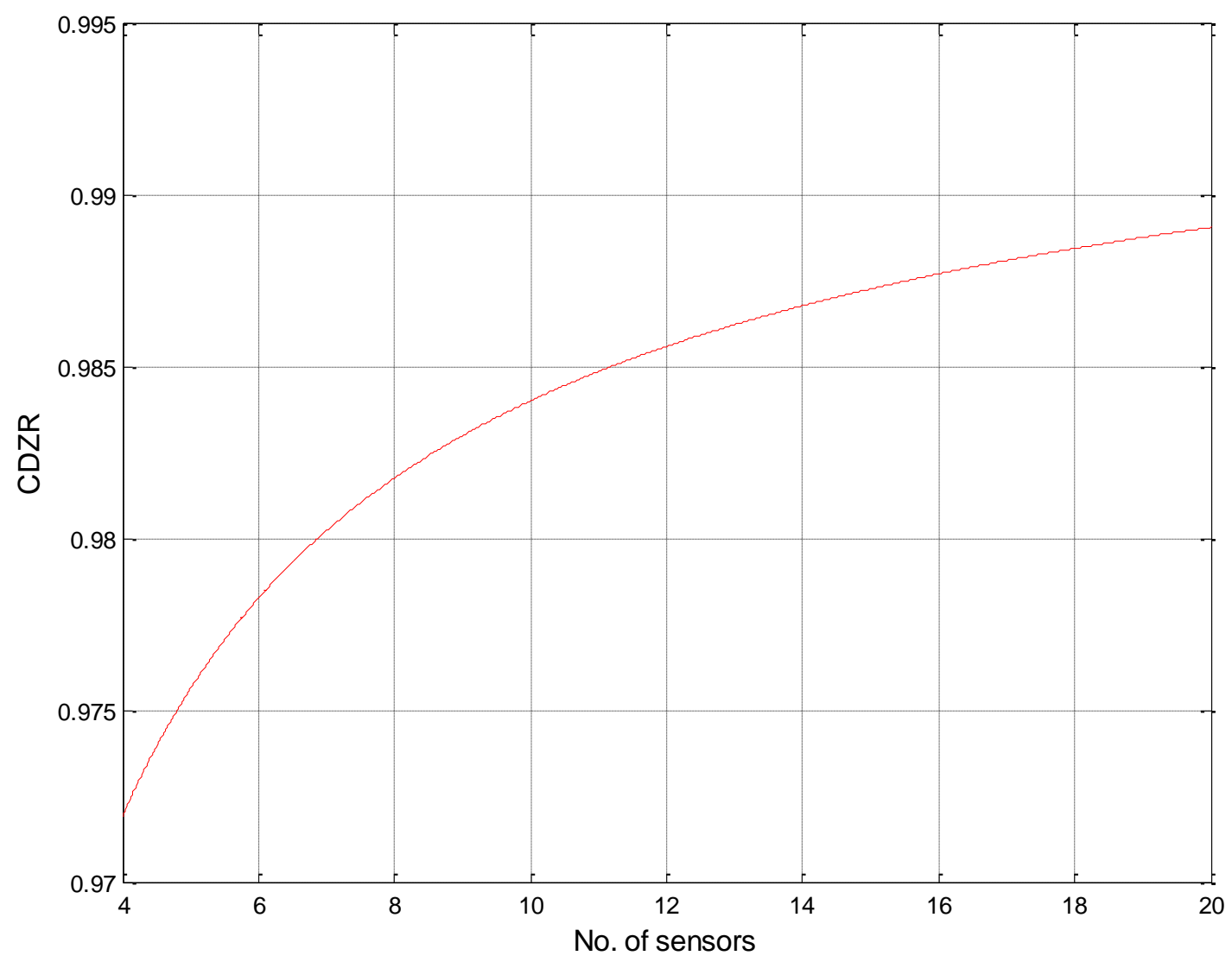

Figure 4-4: Number of sensors versus correct detection zone ratio (CDZR)

$$
\left(\alpha=3.5, l_{0}=38.4 \text { and } \sigma=8\right)
$$

As seen from the previous results, as the number of distributed sensors increase, the accuracy of the constructed REM enhances. The error of the estimated transmitter power decreases, while the correct detection zone ratio increases. The performance starts improving significantly as the number of the distributed sensors increases, but increasing more than 10 sensors achieves slower improvement.

The effect of number of sensors to the CDZR is examined in [51], and they just used 3 different number of sensors 9, 16 and 25 sensors. They concluded that 16 sensors could be the best number of distributed sensors, because the CDZR converges to almost 1 . However, our simulation results consistent with these previous results. We also use three metrics: localization 
error, transmitted signal error and CDZR to demonstrate the effect of increasing the number of sensors from 4 to 20 sensors.

Based on the required accuracy of the system, the optimum number of distributed sensors is selected. The previous presented results are in the best case, where all the measurements and the parameters of the model are accurately correct. However, errors in sensor measurements or in assumed model parameters degrade the performance and we may need more distributed sensors to enhance the quality of the constructed REM.

\subsubsection{Scenario 2: (performance test with different size of hypothetical measurements)}

Because of the effect of shadowing, the measured value of the received signal by each sensor is random. Therefore, an average value of multiple simulated measurements is taken. However, enlarging the sample of measurements causes more delay to construct the REM. Thus, we need to select an appropriate sample size that achieves reasonable accuracy.

Since the matrix form of Eq.(3-25) requires at least four sensors, we assume four sensors displaced randomly in the area of $1 \mathrm{~km}^{2}$ (Figure 4-5). Then, we run the simulation for different number of measurements up to 500 measurements, and each time we test the performance by the presented quality metrics. The considered environment is the same as in the previous scenario. Table 3 summarizes the values of simulation parameters.

(Figure 4-6, Figure 4-7 and Figure 4-8) show the REM performance versus different number of measurements. Since the results have some variations, fitted curves are obtained to represent the scattered results. 
Table 3: Simulation parameters

\begin{tabular}{|cc|}
\hline Parameter & Value \\
\hline Transmitter's actual location & $(400,400)$ \\
\hline Transmitter's actual power & $27 \mathrm{dBm}$ \\
\hline Sensors' locations & 3.5 \\
\hline Path loss exponent $(\boldsymbol{\alpha})$ & $38.4 \mathrm{~dB}$ \\
\hline Path loss constant $\left(\boldsymbol{l}_{\mathbf{0}}\right)$ & $8 \mathrm{~dB}$ \\
\hline Shadowing spread $(\boldsymbol{\sigma})$ & $-114 \mathrm{~dB}$ \\
\hline Minimum detectable signal & \\
\hline
\end{tabular}

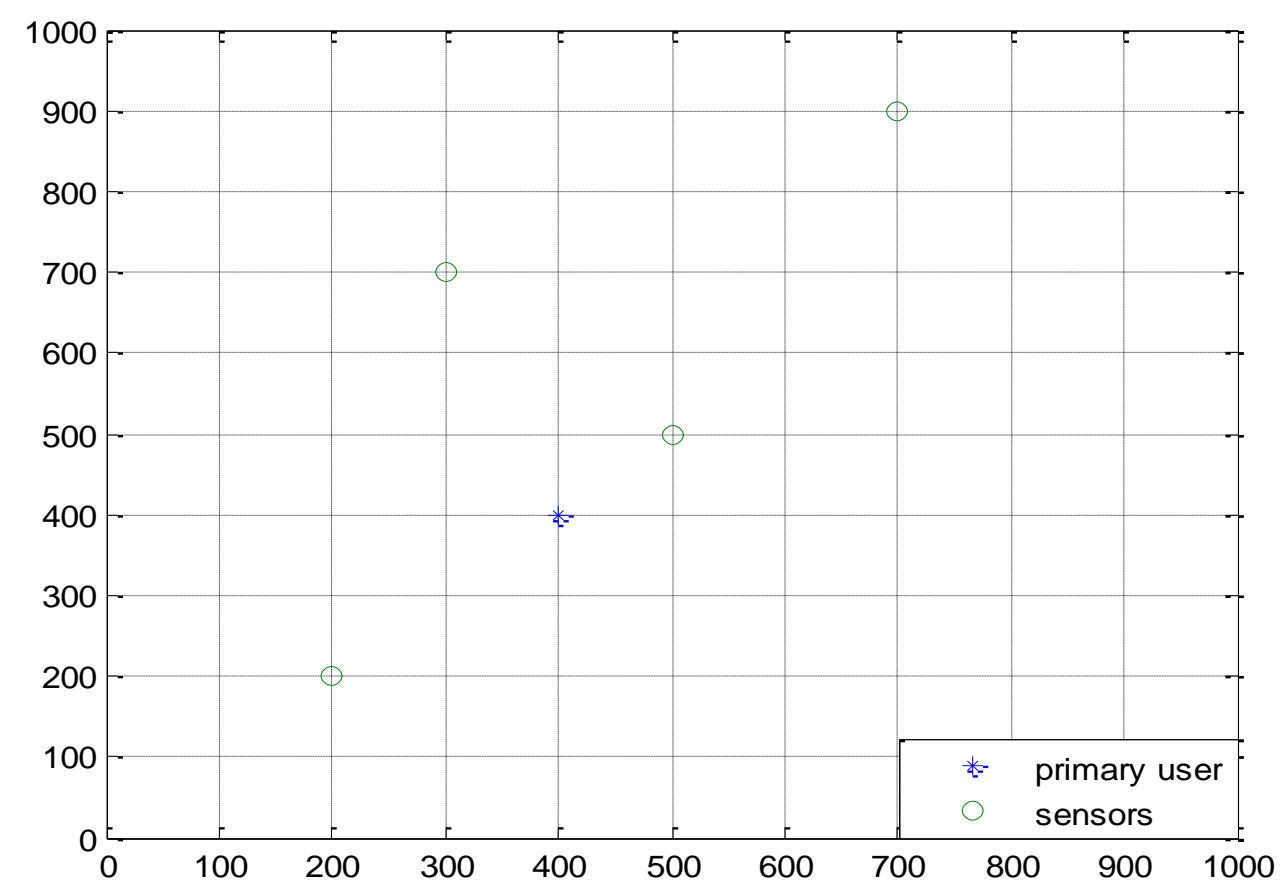

Figure 4-5: Distribution of sensors around the primary user 


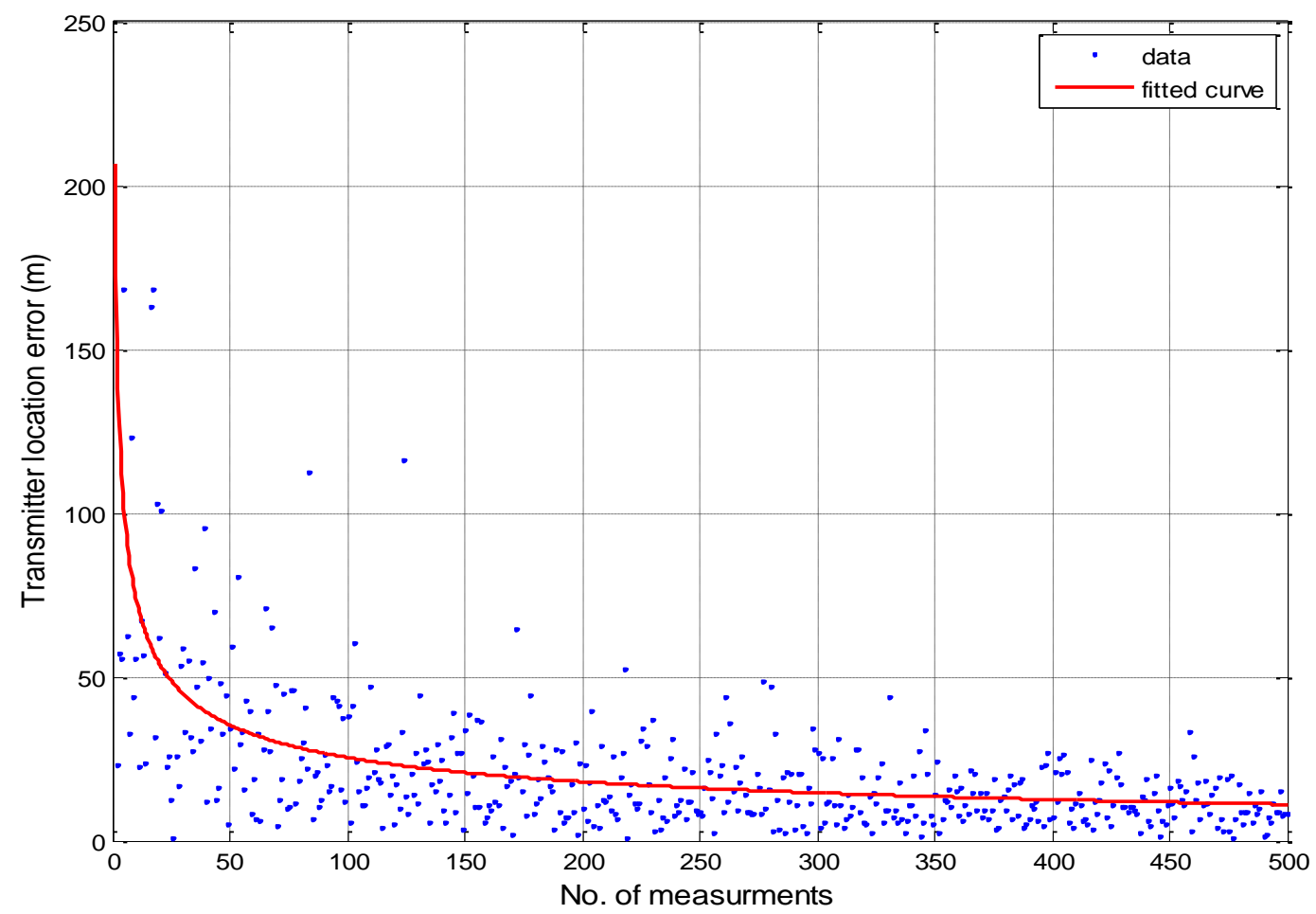

Figure 4-6: Number of hypothetical measurements versus error of the transmitter location $\left(\alpha=3.5, l_{0}=38.4\right.$ and $\left.\sigma=8\right)$

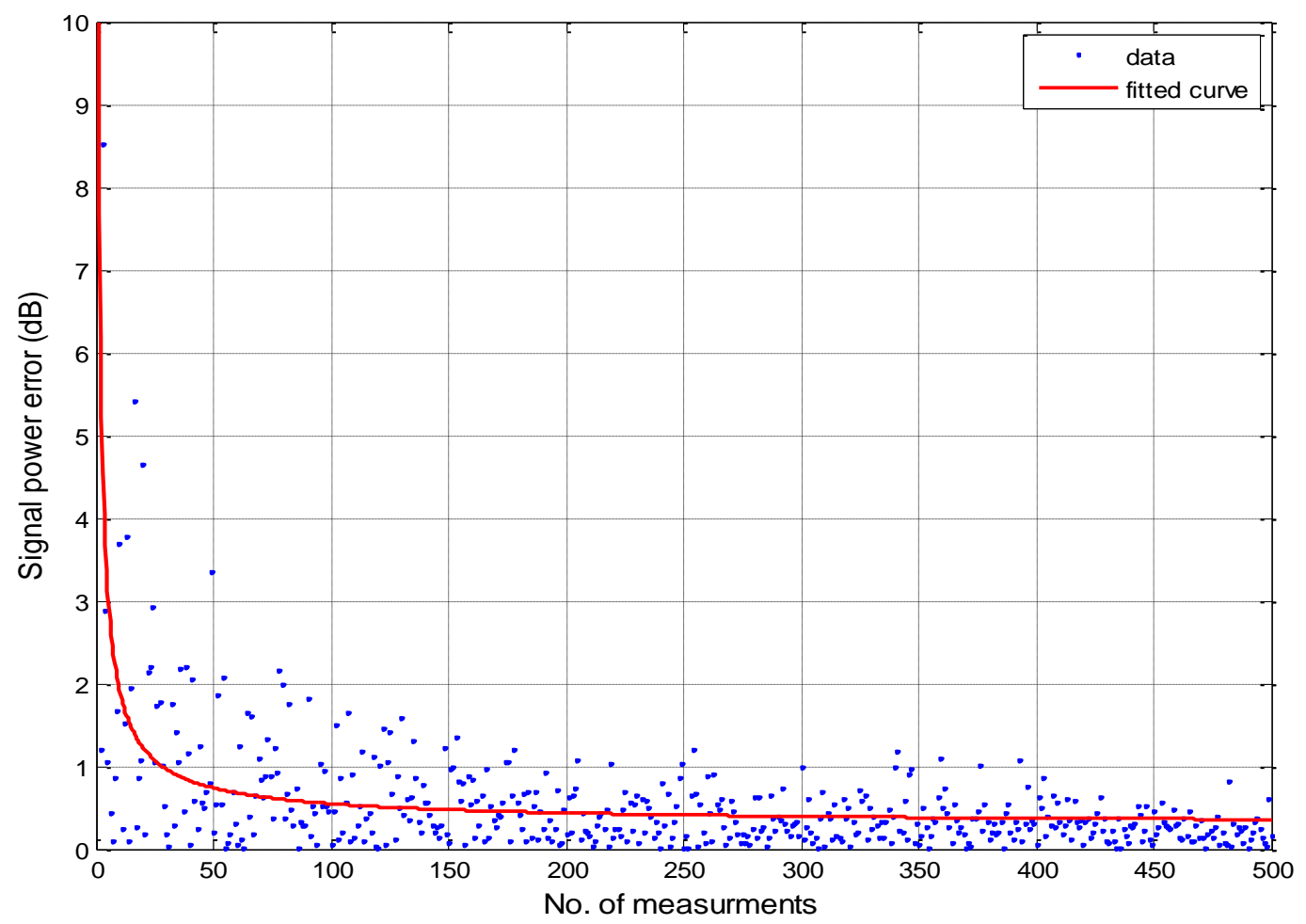

Figure 4-7: Number of hypothetical measurements versus error of the transmitted power $\left(\alpha=3.5, l_{0}=38.4\right.$ and $\left.\sigma=8\right)$ 


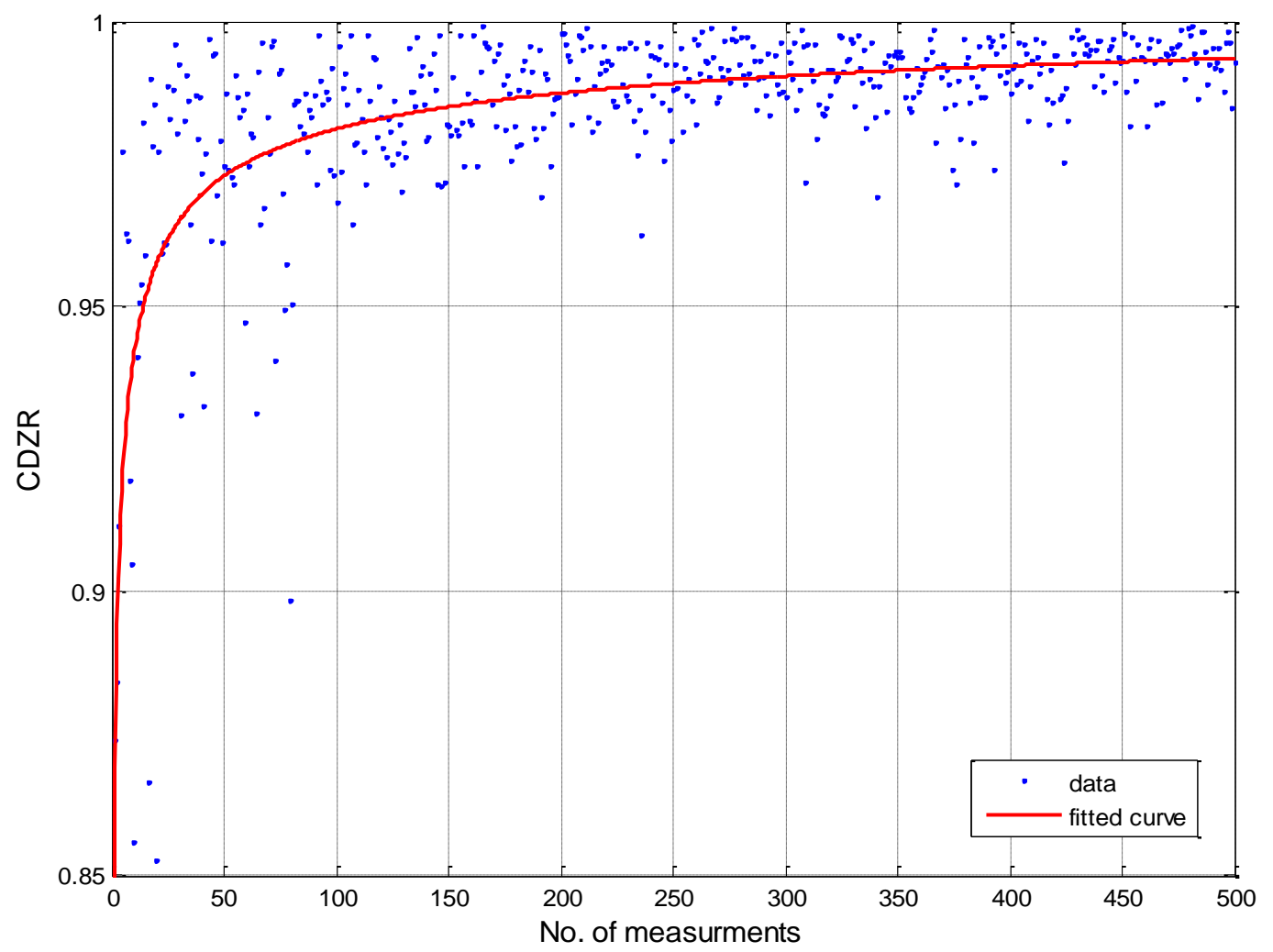

Figure 4-8: Number of hypothetical measurements versus correct detection zone ratio (CDZR)

$$
\left(\alpha=3.5, l_{0}=38.4 \text { and } \sigma=8\right)
$$

It is obvious from the above results that increasing the number of measurements will enhance the accuracy of the estimated values of the transmitter location and power, which will improve the quality of the constructed REM accordingly. Also, increasing the number of measurements will increase the correct detection zone ratio $(C D Z R)$, and as a result, the area of missed detection zone $A\left(Z^{M D}\right)$ is reduced and the lost spectrum chances are lessen. However, as the number of measurements increase rapidly, the accuracy improves slowly. Therefore, based on this simulation results, we see 300 is a reasonable sample size of measurements. Taking larger number of measurements causes more delay in constructing REM process without significant improvement. 
In comparison with previous work, the effect of the number measurement samples was tested in [54] in terms of CDZR. By presenting the results for different number of measurements up to 250 measurements, they concluded that appropriate averaging sample size for RSS can be close to 200, and increasing the number more than that will not improve the performance significantly. Our simulations test the performance using three different quality metrics with a wider range of measurements up to 500 measurements, and reach to a similar conclusion.

\subsubsection{Scenario 3: (performance test under different shadowing spread values)}

In this scenario, we still considering an urban area, but we have uncertainty in the shadow fading standard deviation value. Thus, we need to study the effects of changing standard deviation value to the accuracy of the constructed REM. The simulation has been conducted 10,000 times for values of shadowing standard deviation ranging from 1 to $16 \mathrm{~dB}$. The sample size consists of 300 measurements and the other simulation parameters are the same as illustrated in Table 3. The simulated performance metrics are shown in Figure 4-9, Figure 4-10, and Figure 4-11. 


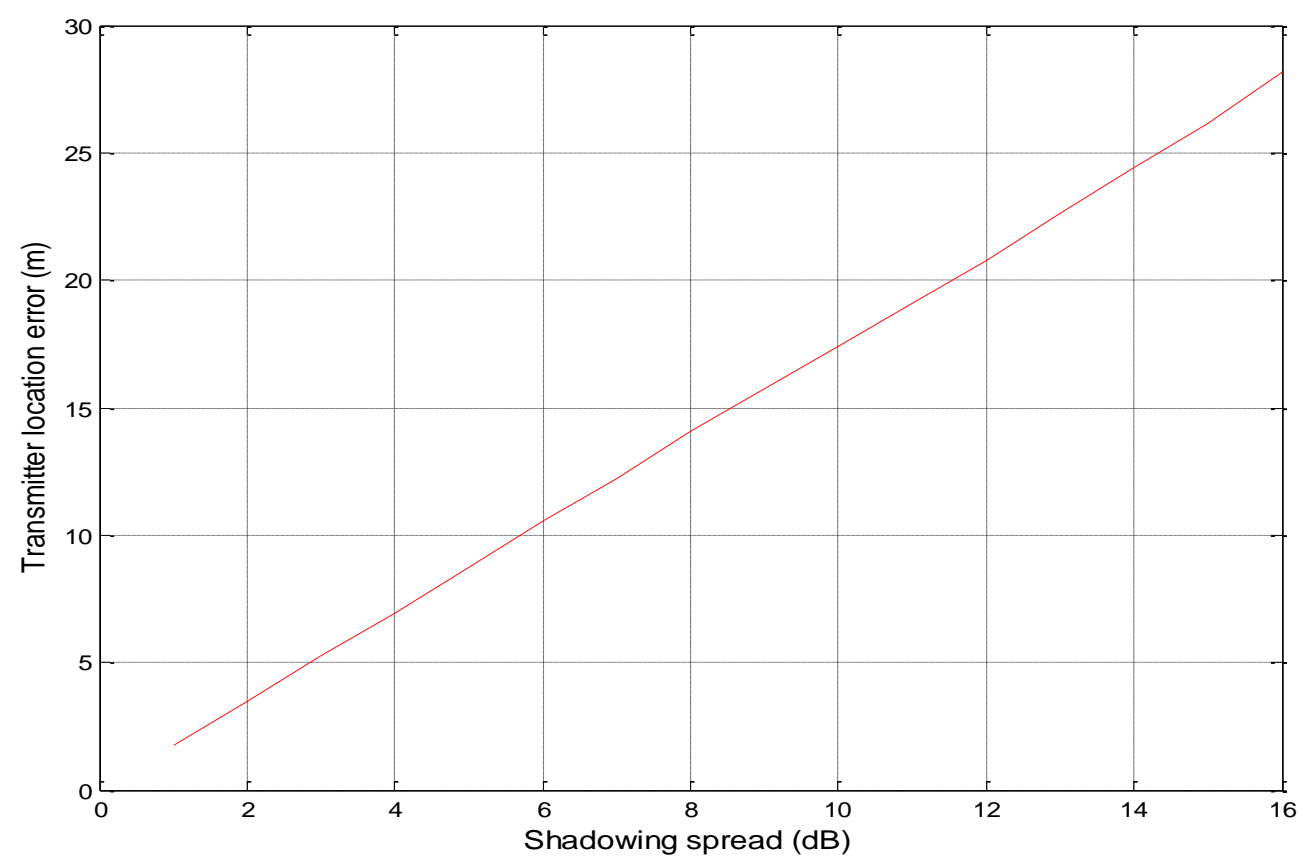

Figure 4-9: Shadow fading standard deviation versus error of the transmitter location $\left(\alpha=3.5, l_{0}=38.4\right.$ and $\left.\sigma=1-16\right)$

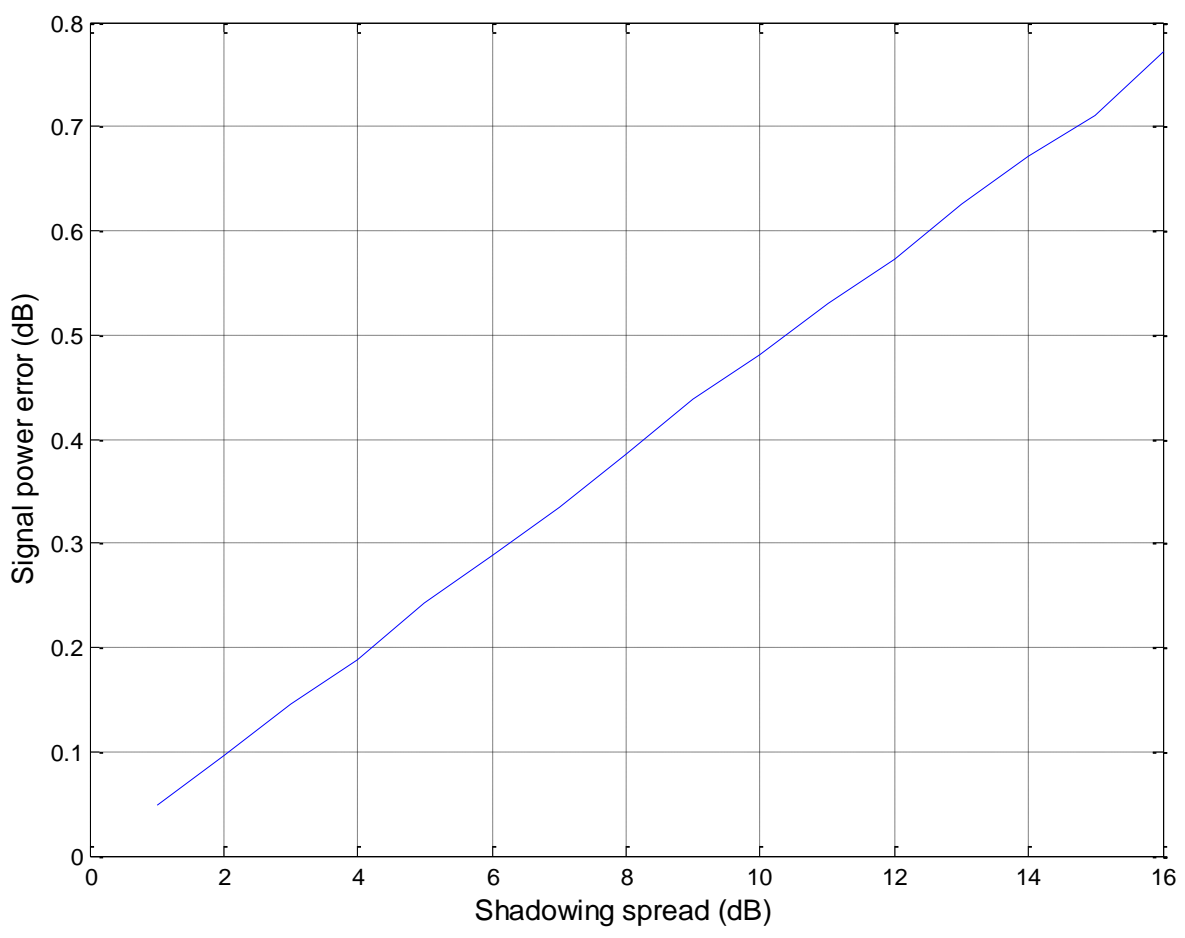

Figure 4-10: Shadow fading standard deviation versus error of the transmitted power $\left(\alpha=3.5, l_{0}=38.4\right.$ and $\left.\sigma=1-16\right)$ 


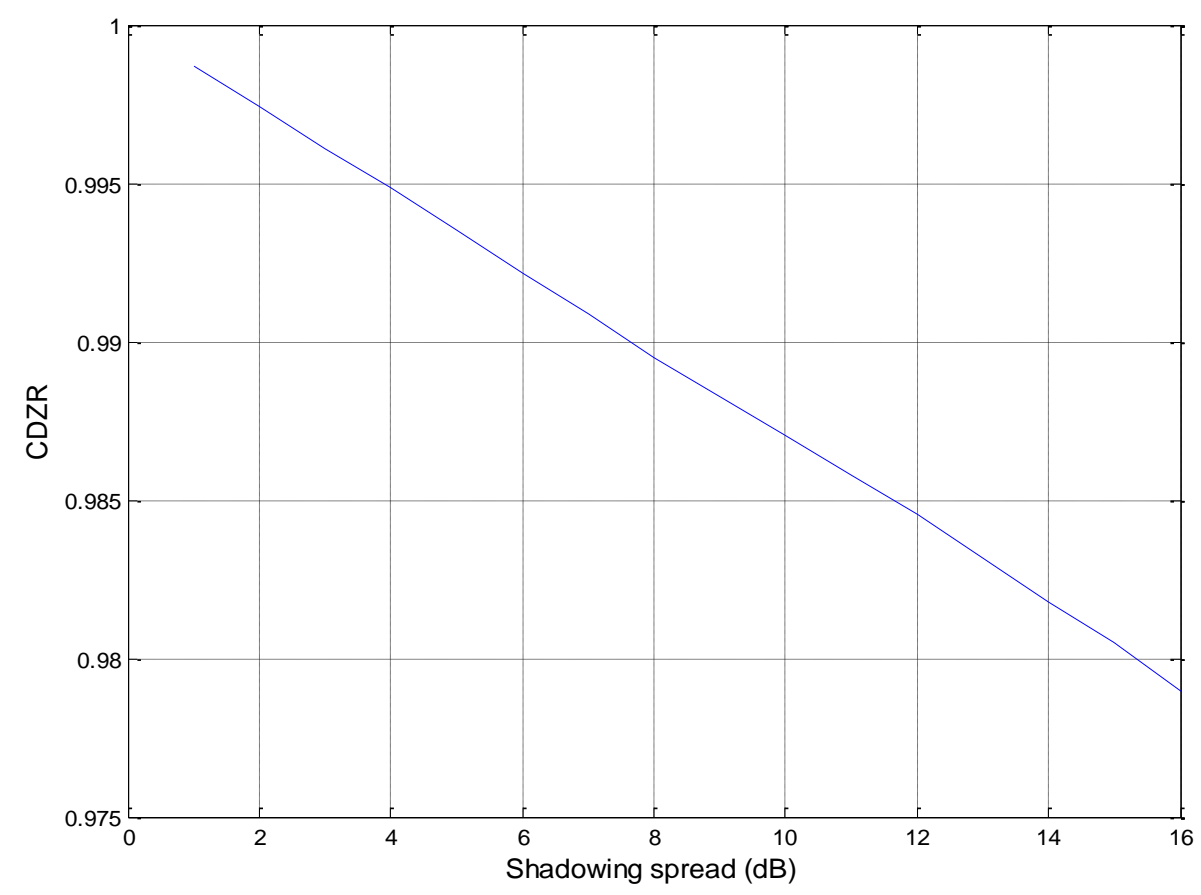

Figure 4-11: Shadow fading standard deviation versus CDZR

$$
\left(\alpha=3.5, l_{0}=38.4 \text { and } \sigma=1-16\right)
$$

From the simulated results, we see that the error of the transmitter parameters increases and the CDZR decreases as the shadowing spread value increases. Therefore, if the environment of the area of interest is subjected to high shadow fading, then we may need to increase the size of the measurement samples and increase the number of distributed sensors in the area to improve the quality of the constructed REM.

\subsubsection{Scenario 4: (performance test with error in path-loss exponent)}

As explained previously, the indirect methods utilize the known model and parameters to construct REM. However, one of the main sources for errors using these methods is inappropriate propagation model or uncalibrated parameters. In this scenario, the considered environment is an urban cellular radio area, where the path-loss exponent $(\boldsymbol{\alpha})$ can be any value in the range $(2.7-3.5)$, as indicated in Table 1 . We assume $(\boldsymbol{\alpha}=3.5)$, but the actual value is 
different from this assumed value by $(5 \%, 10 \%$ or $20 \%)$. Up to the author's knowledge, studying the effects of the errors in estimating path-loss exponent value to the REM performance using this RSS method has not been studied in the literature. Therefore, here is an evaluation of REM in terms of localization error and CDZR metric with different percentages of error in parameter $(\boldsymbol{\alpha})$ and different number of sensors. The number of sensors is ranged from four to twenty sensors, and they are placed randomly in the area. Transmitter's location and other parameters are configured according to Table 3. Localization metric is shown in Figure 4-12, while CDZR metric is depicted in Figure 4-13.

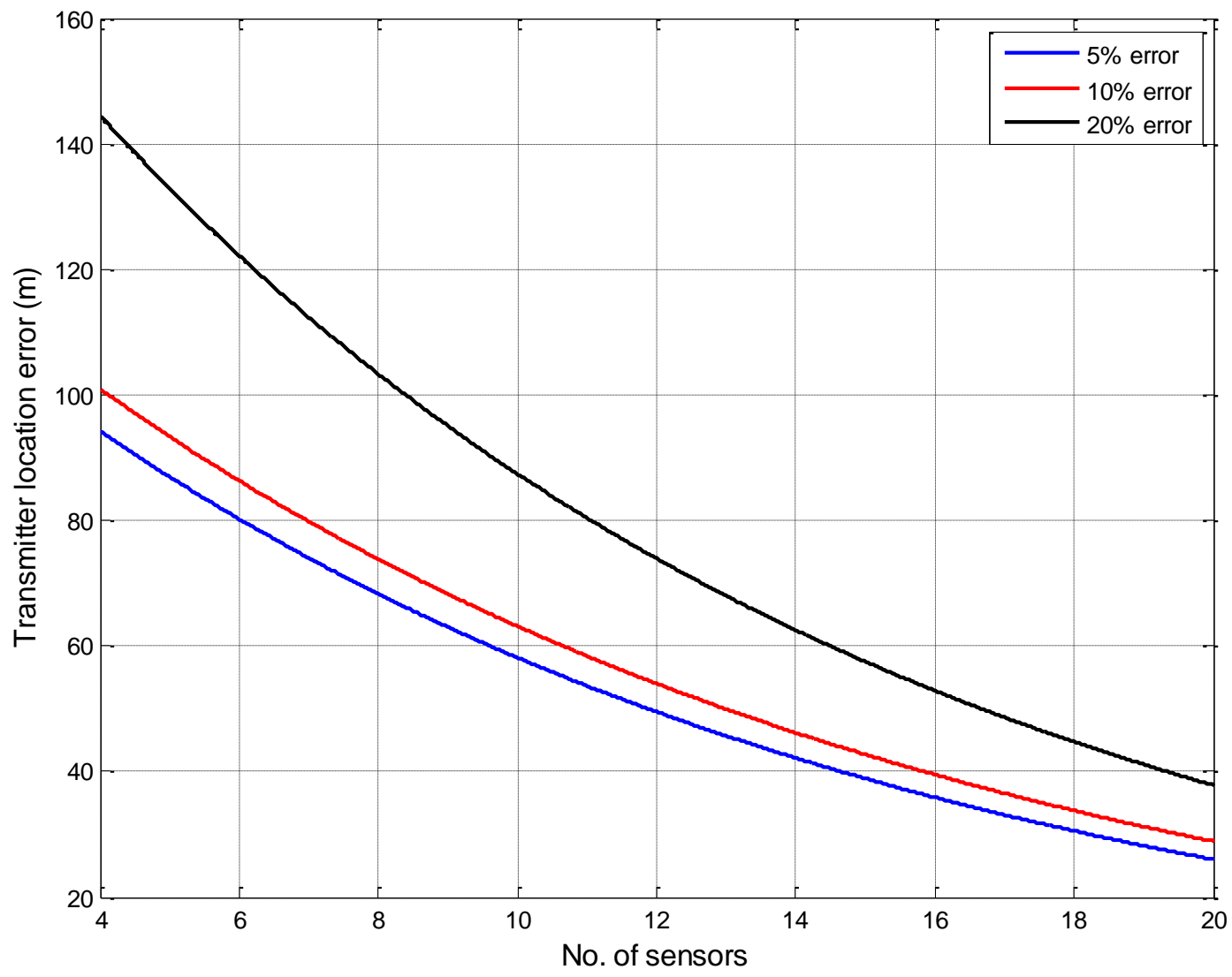

Figure 4-12: Localization error for different percentages of error in $(\alpha)$ and different number of sensors

$$
\left(l_{0}=38.4 \text { and } \sigma=8\right)
$$




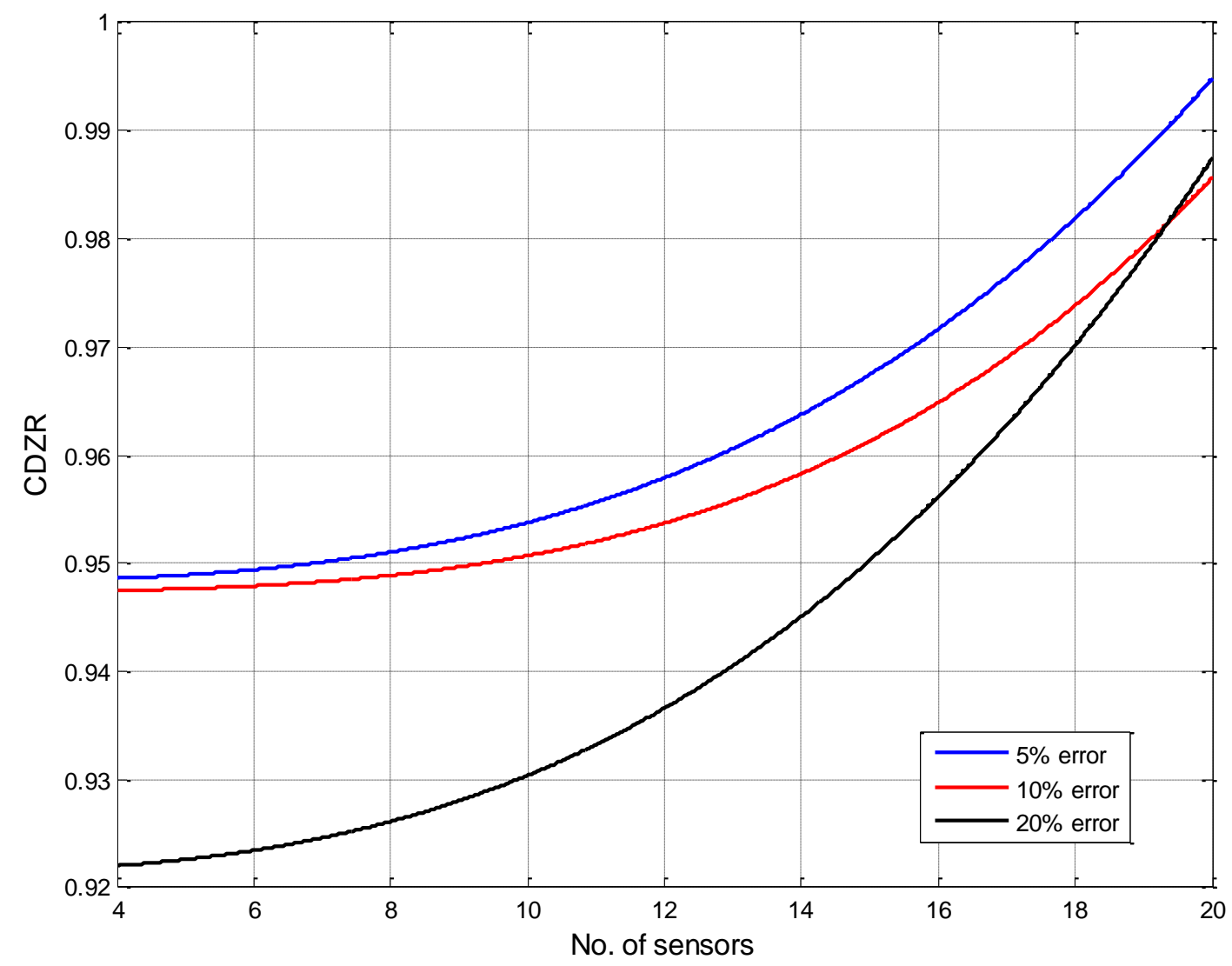

Figure 4-13: CDZR for different percentages of error in $(\alpha)$ and different number of sensors

$$
\left(l_{0}=38.4 \text { and } \sigma=8\right)
$$

The previous results demonstrate that the error in the assumed parameters affect the REM quality significantly. The $5 \%$ and $10 \%$ error of path-loss exponent value have almost similar performance, whereas the quality of the estimated REM degraded substantially as the assumed path-loss exponent value has an error by $20 \%$.

The simulation results also affirm the fact that the REM performance will be enhanced as the number of distributed sensors increases. As seen in Figure 4-12 and Figure 4-13, the worst 
case occurs when we have four sensors and the error of $\alpha$ is $20 \%$. However, as the number of distributed sensors increases or the percentage of error decreases, the performance becomes much better. This simulation scenario tests the sensitivity of the path-loss exponent value, but the results can be generalized to provide insights into expecting similar effects for errors in other assumed model parameters.

\subsubsection{Scenario 5: (REM performance with some moving sensors by a constant speed)}

The previous scenarios dealt with fixed deployed (MCDs). These (MCDs) might be simple measurement sensors, mobile phones, or any similar CR device. However, in some cases secondary users may not have fixed position like a mobile phone user in a moving car. Therefore, we need to simulate the effect of sensors movement to the estimated error of primary transmitter location. We consider different numbers of moving sensors from one to four moving sensors in the area. Up to a total of 20 sensors are distributed randomly in the area of $1 \mathrm{~km}^{2}$ and the number of moving sensors varies from one to four. This movement causes errors in reporting the locations of the sensors. We assume the moving sensors move with a constant speed, and this yields to a $(30 m)$ shift from their actual locations in each REM generation cycle. The movement can be in any direction. Therefore, they might become closer to or far from the transmitter.

Figure 4-14 represents the deployment of sensors in the area and illustrates the movement of the mobile sensors. We consider these sensors $4^{\text {th }}, 8^{\text {th }}, 12^{\text {th }}$ and $16^{\text {th }}$ are mobile secondary users and have motion during REM generation process. The considered environment is an urban area cellular radio and the simulation parameters of the model have been selected according to the same values described in Table 3. Only the localization error metric is used to 
test the performance. A fitted curve of the simulated error of the estimated location of the primary user transmitter versus different numbers of moving sensors is shown in Figure 4-15.

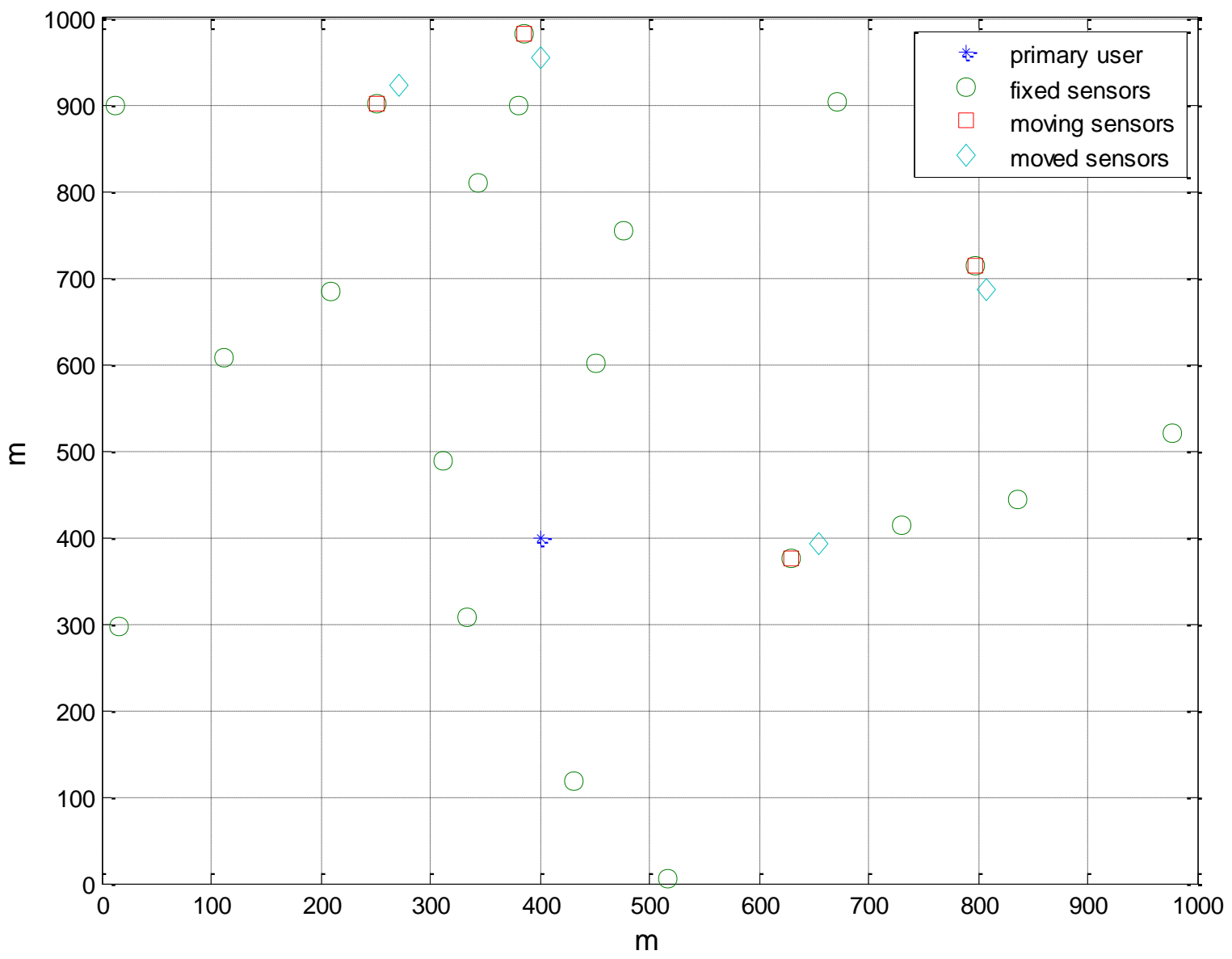

Figure 4-14: Distribution of fixed and mobile moving sensors around the primary user transmitter 


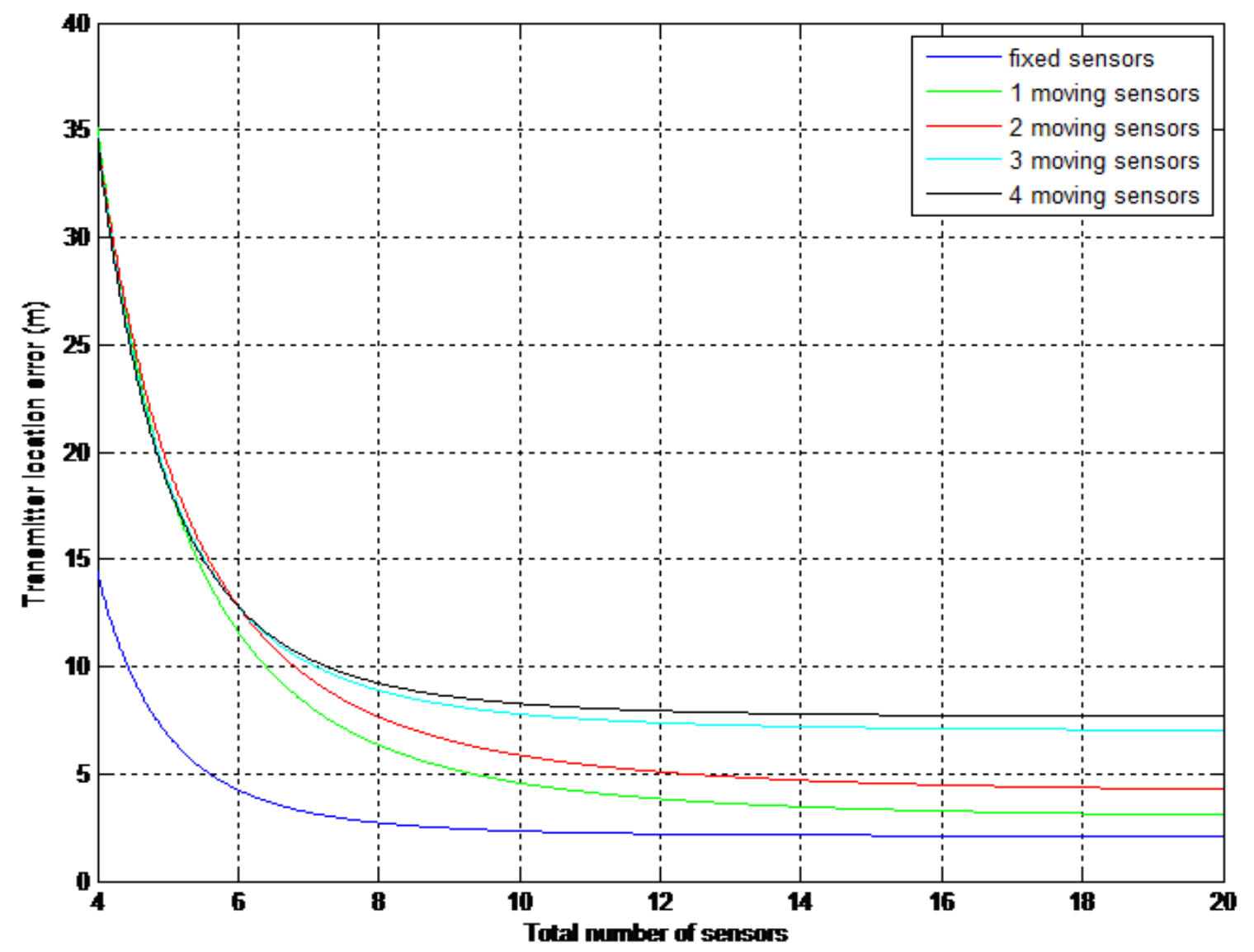

Figure 4-15: Performance comparison for different number of moving sensors versus transmitter location error

Previous simulation result demonstrates that the existence of moving sensors affect the quality of the generated REM. This can be deduced by comparing the fixed sensors curve with the other moving sensors curves. Also, the simulation result clearly shows that as the number of moving sensors increase, the performance and accuracy of REM degrades. In the case of having total of 20 sensors in the area, and up to four moving mobile sensors, we see the worst case happens when we have four moving sensors. The reason for this quality degradation is the movement of the sensors which leads to errors in precisely locating them and accurately measuring the RSS from the transmitter. Therefore, to improve the performance of the constructed REM, we should increase the number of fixed sensors as the number of mobile 
moving sensors increases, in order to mitigate the measurement errors caused by the moving sensors.

\subsubsection{Scenario 6: (REM performance with different speeds of moving sensors)}

In the preceding scenario, our concern was the effect of the number of moving sensors to the accuracy of the generated REM. Here, our interest is to study the effect of the speed of moving sensors to the accuracy of the generated REM. Among the 20 distributed sensors, one sensor is considered as moving sensors, and each simulation curve represents different speed of movement. The sensor that has the mobility feature is selected as the $12^{\text {th }}$ sensor in the order of the displayed results. We assume that we have three levels of speed, and the second and third levels are double and tribble of the first speed. We suppose the first speed causes $30 \mathrm{~m}$ shift for the moving sensor from its location during REM generation. Therefore, the second and third speed can be represented by displacement of $(60 \mathrm{~m}$ and $90 \mathrm{~m})$, respectively. The simulated comparison result using localization metric is shown in Figure 4-16. 


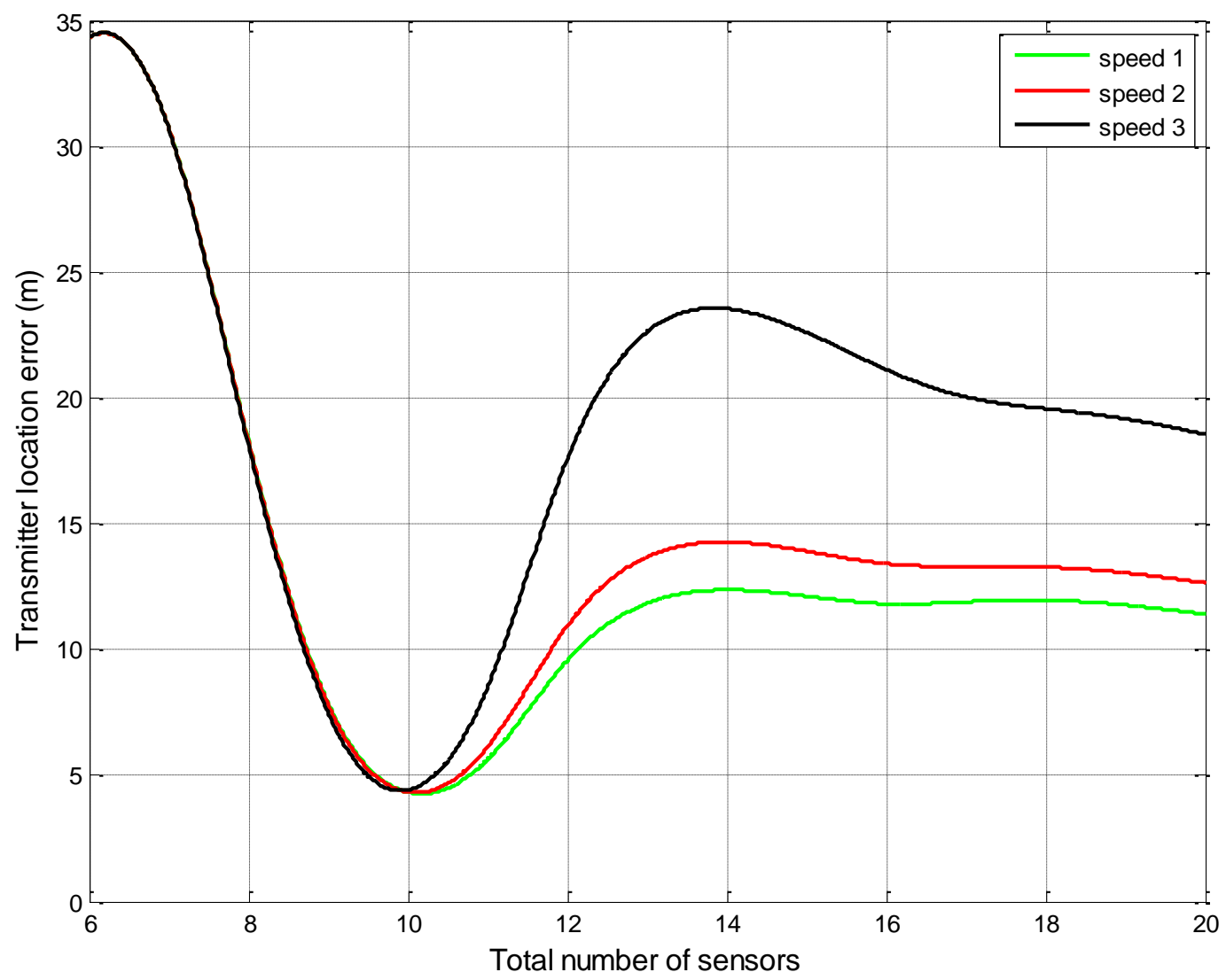

Figure 4-16: Performance comparison of different speeds of moving sensors $12^{\text {th }}$ sensor is moving sensor, (6-11 and 13-20) are fixed sensors

The green curve represents the best performance with the lowest speed of moving sensor, whereas the black one demonstrates the performance of the highest speed which gives the worst quality. The curves in Figure 4-16 are almost identical up to 11 sensors because all these sensors have fixed positions. Since the $12^{\text {th }}$ sensor is selected to have movement feature, the error increases proportionally with speed of movement, and there is about $10 \mathrm{~m}$ difference between the best and the worst case. However, in all curves that represent different speeds, the performance is 
getting much better as the number of fixed sensors increases, as shown clearly in the previous figure in the range (14-20) sensors.

From the previous results, it can be stated that as the speed of moving sensor increases, the estimated transmitter location error increases and REM quality will degrade. However, the performance of all different speeds is enhanced as the number of fixed sensors increases. 


\section{Chapter 5: Conclusions}

This dissertation studies the indirect way for constructing radio environment map (REM) to improve dynamic spectrum access (DSA) in cognitive radio networks (CRNs). Indirect methods are based on localization techniques, which try to utilize known information of the propagation model to estimate the transmitter location and determine the signal level in each location of the area of interest. The first part of this thesis provides the necessary background for REM construction and describes the differences between indirect methods and direct methods, which are based on interpolation techniques. Through the second part, two indirect methods are mathematically presented and their performance is compared, and one of these methods is selected to be tested in different scenarios. The conclusions are presented after each simulation results. This final chapter recaps the previous chapters and summarizes the conclusions of the simulation results. In addition, it proposes some future directions regarding this work.

\subsection{Synthesis of the dissertation:}

Chapter 1 described briefly the concept of CR which has two main features smart cognition and re-configurability. It also provided an overview of the DSA steps in CRNs, which includes spectrum sensing, spectrum management, spectrum mobility and spectrum sharing. The organization of the thesis and a summary of the thesis objectives and contributions were presented at the end of this chapter.

Since REM is a way to improve DSA in CRNs, chapter 2 was devoted to introduce different aspects of REM concept. REM as an integrated database which contains different kinds of information was explained. It also briefly discussed the approaches of acquiring this 
information, and it mentioned three categories of REM application scenarios, which include intra-operator radio resource management, hierarchical spectrum access on licensed bands and spectrum sharing on unlicensed bands. While REM is a wider concept for a multi-layer database, our focus in this thesis was on building the most important layer, the signal interference levels map. The REM designing process and the direct and indirect approaches for constructing REM were briefly explained. An overview of some direct interpolation techniques for constructing REM and several comparative studies between them were presented. Because the indirect approach is a new field in designing REM, and there are some studies that show better performance for indirect methods over direct interpolation methods, indirect approach for constructing REM was selected to be further compared and investigated. The chapter was ended by presenting some metrics that are used to compare between different REM construction techniques.

Chapter 3 described the system models as well as the mathematical analysis of the lognormal shadowing model. Then, two indirect techniques for constructing REM were mathematically presented. The first one uses the RSSD between sensors, whereas the second one is based on RSS values.

Chapter 4 was dedicated for simulating those techniques and test their performance in different cases. For the experiments that have been conducted previously in the literature, we compared our obtained results with previous related work. The comparison between the presented techniques RSSD and RSS showed that in both techniques the accuracy of estimated REM will enhance as the number of distributed sensors increases, but RSS method has better performance RSSD method. However, the RSS method is preferable and reliable if we have a good estimation of path-loss constant value. Based on this result, RSS method was elected for 
further examinations in different scenarios. In the first scenario, the effect of the number of distributed sensors to the accuracy of the generated REM was studied using two metrics signal power error and CDZR. We see that initially as the number of sensors increase, the performance enhances significantly, but later as the number of distributed sensors becomes larger the improvement becomes slow. Second scenario studies the effect of size of measurements to the quality of estimated REM. It is found that 300 is an appropriate averaging sample size of measurement, and this result is compatible with previous work in the literature. The remaining scenarios examine the RSS method in different cases that have not been studied in the literature. In the third scenario, the REM performance under different shadowing spread values was investigated. The simulation results show that increasing the shadowing spread values degrades the quality of the estimated REM. Therefore, if the area of interest is subject to high shadowing, we expect large errors and we may need more distributed sensors to mitigate the shadowing effect. The forth scenario studies the effect of the accuracy of estimated model parameters to the quality of the estimated REM. Specifically, different percentages of error in estimated path-loss exponent $(\boldsymbol{\alpha})$ to the performance of REM have been studied. The results showed that as the percentage of possible error in estimated path-loss exponent increases, the REM quality is affected significantly. Therefore, uncalibrated parameters or inappropriate propagation model will degrade the performance substantially, and the indirect method for constructing REM may become ineffective. In the fifth and sixth scenario, an environment that contains a mixture of fixed and mobile sensors was considered, and the effect of the number of moving sensors and their speed to the REM quality was studied. It was shown that increasing the number of moving sensor or their speed, the error of the estimated transmitter location will increase and subsequently the REM performance will degrade. However, some improvements in the 
generated REM can be achieved by increasing the number of fixed sensors to mitigate the error caused by moving sensors.

\subsection{Future work:}

There are many topics that deserve further investigations. Here is a list of some interesting directions.

- In several practical situations, we have multiple primary users. Therefore, constructing REM in an environment with multiple transmitters merits further study.

- Random distribution of sensors was considered in this dissertation. The effect of using uniform deployment of sensors to the REM quality can be examined.

- Updating REM information more frequently makes the system more complex, but if the network is more dynamic and the updates are less frequently, the REM information may become ineffective. Therefore, deciding the optimum update intervals that regard the tradeoff between the REM accuracy and the complexity of the system is yet to be explored. 


\section{References}

[1] J. Mitola III, “Cognitive Radio for Flexible Mobile Multimedia Communications,” in IEEE International Workshop on Mobile Multimedia Communications, 1999, pp. 3-10.

[2] J. Mitola III, “Cognitive Radio An Integrated Agent Architecture for Software Defined Radio,” Royal Institute of Technology (KTH), Sweden, 2000.

[3] S. Haykin, "Cognitive Radio : Brain-Empowered Wireless Communications,” IEEE J. Sel. Areas Commun., vol. 23, no. 2, pp. 201-220, 2005.

[4] M. A. Mchenry, P. A. Tenhula, D. A. Roberson, and C. S. Hood, "Chicago Spectrum Occupancy Measurements \& Analysis and a Long-term Studies Proposal," in Proceedings of the First International Workshop on Technology and policy for accessing spectrum, 2006.

[5] M. Nekovee, "Quantifying the Availability of TV White Spaces for Cognitive Radio Operation in the UK," in Proceedings of the IEEE International Conference on Communications, 2009.

[6] I. F. Akyildiz, W. Lee, M. C. Vuran, and S. Mohanty, “A survey on spectrum management in cognitive radio networks," Cogn. RADIO Commun. NETWORKS, no. April, pp. 40-48, 2008.

[7] I. F. Akyildiz, W. Lee, M. C. Vuran, and S. Mohanty, "NeXt generation / dynamic spectrum access / cognitive radio wireless networks : A survey," Comput. Networks, vol. 50, pp. 2127-2159, 2006.

[8] F. F. Digham, M. Alouini, S. Member, and M. K. Simon, "On the Energy Detection of Unknown Signals Over Fading Channels,” IEEE Trans. Commun., vol. 55, no. 1, pp. 21- 
$24,2007$.

[9] P. D. Sutton, K. E. Nolan, and L. E. Doyle, "Cyclostationary Signatures in Practical Cognitive Radio Applications," IEEE J. Sel. Areas Commun., vol. 26, no. 1, pp. 13-24, 2008.

[10] H. Tang, "Some Physical Layer Issues of Wide-band Cognitive Radio Systems," in Proc. IEEE Int. Symposium on New Frontiers in Dynamic Spectrum Access Networks, 2005, pp. $151-159$.

[11] H. Yu, Y. Sung, and Y. H. Lee, “On optimal operating characteristics of sensing and training for cognitive radios," in Proceedings of IEEE International Conference on Acoustics, Speech, and Signal Processing, 2008, pp. 2785-2788.

[12] T. Yucek and H. Arslan, "A Survey of Spectrum Sensing Algorithms for Cognitive Radio Applications,” IEEE Commun. Surv. Tutorials, vol. 11, no. 1, pp. 116-130, 2009.

[13] A. M. Wyglinski, M. Nekovee, and T. Hou, Cognitive Radio Communications and Networks Principles and Practice. Academic Press, 2010.

[14] I. F. Akyildiz, B. F. Lo, and R. Balakrishnan, "Cooperative spectrum sensing in cognitive radio networks : A survey," Phys. Commun., vol. 4, no. 1, pp. 40-62, 2011.

[15] W. Zhang, R. K. Mallik, and K. B. Letaief, “Cooperative Spectrum Sensing Optimization in Cognitive Radio Networks," in IEEE International Conference on Communications, 2008, pp. 3411-3415.

[16] W. Krenik and A. Batra, "Cognitive radio techniques for wide area networks," in 42nd Design Automation Conference, 2005. Proceedings, 2005, pp. 409-412.

[17] Y. Zhao, B. Le, and J. H. Reed, "Network Support: The Radio Environment Map," in Cognitive Radio Technology, 2nd ed., B. Fette, ed. Academic Press, 2008. 
[18] Y. Zhao, "Enabling Cognitive Radios through Radio Environment Maps," PhD dissertation submitted to the Faculty of the Virginia Polytechnic Institute and State University, 2007.

[19] M. Ibnkahla, Cooperative Cognitive Radio Networks: The Complete Spectrum Cycle. CRC Press, 2014.

[20] FCC, "Federal Communications Commissions,47 CFR Parts 0 and 15 Unlicensed Operation in the TV Broadcast Bands; Final Rule," 2015.

[21] "Flexible and Spectrum Aware Radio Access through Measurements and Modelling in Cognitive Radio Systems FARAMIR, Document Number D4 . 1, Radio Environmental Maps: Information Models and Reference Model,” Measurement, pp. 1-73, 2011.

[22] H. Yilmaz Birkan, T. Tugcu, F. Alagöz, and S. Bayhan, "Radio environment map as enabler for practical cognitive radio networks," IEEE Commun. Mag., vol. 51, no. 12, pp. $162-169,2013$.

[23] Standard for Wireless Regional Area Networks (WRAN)—Specific requirements, Part 22: Cognitive Wireless RAN Medium Access Control (MAC) and Physical Layer (PHY) Specifications: Policies and Procedures for Operation in the TV Bands, IEEE 802.22. 2011.

[24] Y. Zhao, L. Morales, J. Gaeddert, K. K. Bae, J. S. Um, and J. H. Reed, “Applying radio environment maps to cognitive wireless regional area networks," in 2007 2nd IEEE International Symposium on New Frontiers in Dynamic Spectrum Access Networks, 2007, pp. $115-118$.

[25] J. Zhang, Z. Qi, and J. Zou, “The IEEE802.22 WRAN system based on Radio Environment Map (REM)," Proc. 1st Int. Work. Educ. Technol. Comput. Sci. ETCS 2009, 
vol. 1, pp. 98-101, 2009.

[26] S. Subramani, B. Sayrac, L. Gavrilovska, M. Sooriyabandara, T. Farnham, and O. Labs, “Towards Practical REM-based Radio Resource Management," in Future Network \&MobileSummit, 2011, pp. 1-8.

[27] S. Eberly, J. Swall, D. Holland, B. Cox, and E. Baldridge, "Developing Spatially Interpolated Surfaces and Estimating Uncertainty, Publication No. EPA-454/R-04-004,” 2004.

[28] E. Dall'Anese, S. J. Kim, and G. B. Giannakis, “Channel gain map tracking via distributed kriging," IEEE Trans. Veh. Technol., vol. 60, no. 3, pp. 1205-1211, 2011.

[29] K. Sato and T. Fujii, "Kriging-based Interference Power Constraint for Spectrum Sharing based on Radio Environment Map,” in IEEE Globecom Workshops (GC Wkshps), 2015.

[30] K. Rohr, "Landmark-based image analysis: using geometric and intensity models," in Computational Imaging and Vision Series, 2001.

[31] G. Mateos, J.-A. Bazerque, and G. B. Giannakis, "Spline-based spectrum cartography for cognitive radios," in Conference Record - Asilomar Conference on Signals, Systems and Computers, 2009, no. 1, pp. 1025-1029.

[32] C. Harman and M. Johns, "Voronoi Natural Neighbors Interpolation."

[33] L. Bolea, J. Pérez-romero, and R. Agustí, "Received Signal Interpolation for Context Discovery in Cognitive Radio," in 14th Int. Symposium on Wireless Personal Multimedia Communications (WPMC), 2011.

[34] S. Üreten, A. Yongaçoglu, and E. Petriu, “A Comparison of Interference Cartography Generation Techniques in Cognitive Radio Networks," in IEEE International Conference on Communications (ICC), 2012. 
[35] M. Pesko, T. Javornik, A. Košir, M. Štular, and M. Mohorčič, "Radio environment maps: The survey of construction methods," KSII Trans. Internet Inf. Syst., vol. 8, no. 11, pp. 3789-3809, 2014.

[36] L. Bolea, J. Pérez-Romero, R. Agustí, and O. Sallent, "Context discovery mechanisms for cognitive radio," IEEE 73rd Veh. Technol. Conf. (VTC2011 Spring), 2011.

[37] D. Denkovski, V. Atanasovski, L. Gavrilovska, J. Riihijaervi, and P. Maehoenen, "Reliability of a Radio Environment Map: Case of Spatial Interpolation Techniques," Proc. 7th Int. Conf. Cogn. Radio Oriented Wirel. Networks, 2012.

[38] M. A. and K. Dos Ramos, “A Comparison of Spatial Interpolation Methods for Estimation of Average Electromagnetic Field Magnitude," Prog. Electromagn. Res.M(PIERM), vol. 14, no. September, pp. 135-145, 2010.

[39] M. Angjelicinoski, V. Atanasovski, and L. Gavrilovska, "Comparative analysis of spatial interpolation methods for creating Radio Environment Maps," in 19th Telecommunications Forum (TELFOR), 2011, pp. 334-337.

[40] S. Üreten, A. Yongaçoglu, and E. Petriu, “Interference Map Generation Based on Delaunay Triangulation in Cognitive Radio Networks," in IEEE 13th International Workshop on Signal Processing Advances in Wireless Communications (SPAWC), 2012, pp. $134-138$.

[41] J. D. Naranjo, A. Ravanshid, I. Viering, and G. Bauch, "Interference Map Estimation Using Spatial Interpolation of MDT Reports in Cognitive Radio Networks," in IEEE Wireless Communications and Networking Conference (WCNC), 2014.

[42] M. Pesko, T. Javornik, L. Vidmar, A. Košir, M. Štular, and M. Mohor`, "The indirect selftuning method for constructing radio environment map using omnidirectional or 
directional transmitter antenna," EURASIP J. onWireless Commun. Netw., 2015.

[43] K. S. (RMIT) Wei Jiang, Hanwen Cao, Michael Wielemer(UDE) Tomaž Javornik, Igor Ozimek, Marko Pesko, Matevž Vučnik (JSI) Nishantha Hettiarachi, Shweta Jain, "Performance of Spectrum Awareness Techniques, Integrated Project ABSOLUTE Aerial Base Stations with Opportunistic Links for Unexpected \& Temporary Events," 2014.

[44] G. Mao, B. Fidan, and B. D. O. Anderson, "Wireless sensor network localization techniques," Comput. Networks, vol. 51, pp. 2529-2553, 2007.

[45] N. Patwari, J. N. Ash, S. Kyperountas, A. O. H, R. L. Moses, and N. S. Correal, "Locating the Nodes [Cooperative localization in wireless sensor networks]," IEEE Signal Process. Mag., no. July, pp. 54-69, 2005.

[46] A. Roxin, J. Gaber, M. Wack, and A. Nait-Sidi-Mo, "Survey of Wireless Geolocation Technique," in IEEE Globecom Workshops, 2007.

[47] S. Üreten, A. Yongaçoglu, and E. Petriu, "Iterative Grid Search for RSS-Based Emitter Localization," in 22nd European Signal Processing Conference (EUSIPCO), 2014, pp. 1507-1511.

[48] S. Üreten, A. Yongaçoglu, and E. Petriu, “A Reduced Complexity Iterative Grid Search for RSS-Based Emitter Localization," in 27th Biennial Symposium on Communications (QBSC), 2014, pp. 149-152.

[49] S. Üreten, A. Yongaçoglu, and E. Petriu, "Primary emitter localization using smartly initialized Metropolis-Hastings algorithm," in Proceedings of the 21st European Signal Processing Conference (EUSIPCO), 2013, pp. 1-5.

[50] B. R. Jackson, S. Wang, and R. Inkol, "Received signal strength difference emitter 
geolocation Least Squares algorithm comparison," in Canadian Conference on Electrical and Computer Engineering (CCECE), 2011.

[51] H. B. Yilmaz and T. Tugcu, "Location estimation-based radio environment map construction in fading channels," Wirel. Commun. Mob. Comput., pp. 421-430, 2015.

[52] T. Rappaport, Wireless Communications Principles andPractice, Second. Prentice Hall, 2013.

[53] "Final Report on Link Level and System Level Channel Models, IST-2003-507581 WINNER, D5.4 v. 1.4,” 2005.

[54] H. B. Yilmaz, "Cooperative Spectrum Sensing and Radio Environment Map Construction in Cognitive Radio Networks," PhD thesis in Bogazici University, Istanbul, Turkey, 2012. 